\title{
Outcomes in adult pectus excavatum patients undergoing Nuss repair
}

This article was published in the following Dove Press journal: Patient Related Outcome Measures

\section{MennatAllah M Ewais \\ Shivani Chaparala \\ Rebecca Uhl \\ Dawn E Jaroszewski}

Department of Cardiothoracic Surgery, Mayo Clinic Hospital, Phoenix, AZ, USA
Correspondence: Dawn E Jaroszewski Department of Cardiothoracic Surgery, Mayo Clinic Hospital, 5777 E Mayo Blvd, Phoenix, AZ 85054, USA

Tel +l 4803422270

$\mathrm{Fax}+\mathrm{I} 4803422269$

Email jaroszewski.dawn@mayo.edu

\begin{abstract}
Pectus excavatum (PEx) is one of the most common congenital chest wall deformities. Depending on the severity, presentation of PEx may range from minor cosmetic issues to disabling cardiopulmonary symptoms. The effect of PEx on adult patients has not been extensively studied. Symptoms may not occur until the patient ages, and they may worsen over the years. More recent publications have implied that PEx may have significant cardiopulmonary implications and repair is of medical benefit. Adults presenting for PEx repair can undergo a successful repair with a minimally invasive "Nuss" approach. Resolution of symptoms, improved quality of life, and satisfying results are reported.
\end{abstract}

Keywords: complications, minimally invasive surgery, quality of life

\section{Background}

Pectus excavatum (PEx) is the most common congenital chest wall anomaly. ${ }^{1-4}$ The deformity is reported to occur more frequently in males than females; however, diagnosis in females may often be missed if obscured with breast tissue. ${ }^{5-7}$ Depending on the severity, presentation of PEx may range from a minor cosmetic issue to disabling cardiopulmonary symptoms. ${ }^{8-10}$ The internally displaced sternum can cause right-side heart compression and restrictive deficits. ${ }^{8,9}$ As the patient advances in age, the chest wall can become less flexible as a result of increased calcium accumulation in cartilage attachments of the anterior chest wall. ${ }^{11,12}$ Symptoms may occur or show progression as the patient ages. ${ }^{11-13}$ Kragten et $\mathrm{al}^{12}$ reported development of symptoms in the fourth and fifth decade in nearly half of their adult patients with significant improvement after surgical repair. The optimal surgical procedure for adult PEx patients has been controversial, and some surgeons recommend limiting the Nuss procedure to pediatrics and adolescents. ${ }^{14,15}$ We present a review of adult patients with PEx including outcomes after repair with Nuss ("Nuss") or a minimally invasive repair of pectus excavatum (MIRPEx).

\section{Cardiopulmonary outcomes}

The cardiopulmonary effects of PEx have been debated for years, ${ }^{16-18}$ and there is a paucity of reports evaluating adult patients. ${ }^{19,20}$ The inward deformity of the anterior chest wall has a negative cardiopulmonary consequence on patients with PEx, as supported by the most recent data. ${ }^{9,21}$ This can cause displacement of the heart into the left chest and varying degrees of heart compression (Figure 1). Decrease in atrial filling and venous return can result in diastolic dysfunction and reduction in cardiac 
output with significant compression to the chambers of the right heart. ${ }^{9,20-22}$ Mocchegiani et $\mathrm{al}^{23}$ reported that the right ventricular outflow tract in PEx patients was significantly narrower and right ventricle (RV) end-diastolic and-systolic areas were significantly smaller. Surgical correction of the PEx has been shown to relieve compression, allowing for a significant increase in right heart chamber size, increased flow velocities, and improved cardiac output ${ }^{9,21,24}$ (Figure 2A and B). Krueger et $\mathrm{al}^{25}$ also noted significant improvement in post-repair cardiac outputs that increased to $66.2 \%$ vs $58.4 \%$ and the end-diastolic RV volume that increased to a mean of $40.8 \mathrm{~mL}$ vs $21.7 \mathrm{~mL}$ preoperatively. There may be a greater impact on cardiac function and symptoms in patients over 30 years of age. ${ }^{12,24}$ In post-repair PEx patients $\geq 30$ years, a mean increase in right ventricular output of $65 \%$ was documented

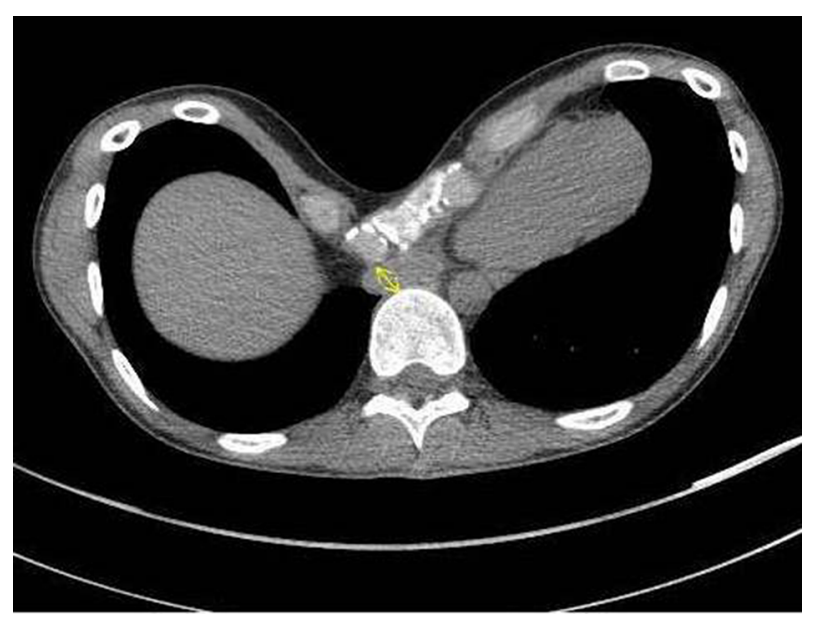

Figure I Computerized tomographic scan of a patient with severe pectus excavatum and Haller index of 24.6. Sternal deformity with compression of the right heart and inflow are seen (arrow). by intraoperative transesophageal echocardiogram. ${ }^{24}$ Neviere et $\mathrm{al}^{26}$ found that PEx deformity was associated with reduction in the strength of the inspiratory muscle as evident by reduction in the maximal static respiratory pressure (PImax) and sniff nasal inspiratory pressure (SNIP) with significant increase of these values postoperatively which was reflected in enhanced efficacy of the respiratory pump and the cardiovascular function improvement.

Long-term follow-up of corrected PEx patients and correlations between physiologic impact and symptoms are lacking. ${ }^{8,27-30}$ Table 1 reviews some of the major publications reporting cardiopulmonary impairments and postsurgical results. Only six of these represented a mean age of 18 years and older. ${ }^{9,19,25,26,31,32}$ Cardiopulmonary exercise testing has been used to assess exercise capacity and limitations in peak oxygen uptake and $\mathrm{O}_{2}$ pulse which can result as a consequence of the pectus deformity. ${ }^{18,19,33,34}$ Publications as to the benefits of surgical correction have varied. $8,19,31,35$ Several studies have reported that the cardiopulmonary function has improved significantly with increase in the oxygen consumption $\left(\mathrm{VO}_{2}\right)$ and $\mathrm{O}_{2}$ pulse after surgical repair of $\mathrm{PEx} .^{8,19}$ Maagaard et $\mathrm{al}^{35}$ previously reported normalization of the decreased cardiopulmonary function in teenagers with PEx at 3 years following surgical repair; however, in a more recent evaluation of adult patients by Udholm et $\mathrm{al}^{31}$ ( $\geq 21$ years), a significant improvement in the maximum oxygen consumption $\left(\mathrm{VO}_{2} \max \right)$ was not seen 1 year after PEx repair. These results did show a trend of increased improvement in the $\mathrm{VO}_{2}$ max which could be more evident with a longer period of follow-up. Adult patients may also differ in their ability to return to normal after PEx repair. During assessment, the patient's baseline exercise history must be considered.
A

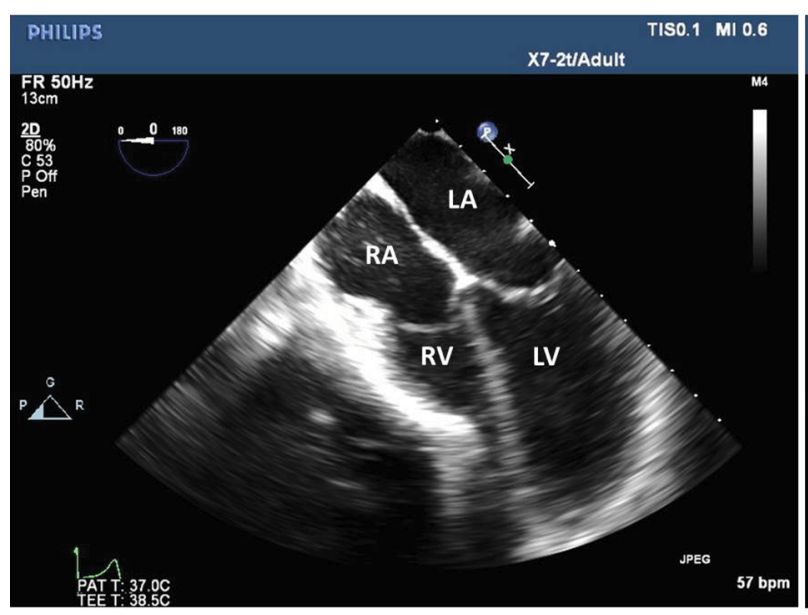

B

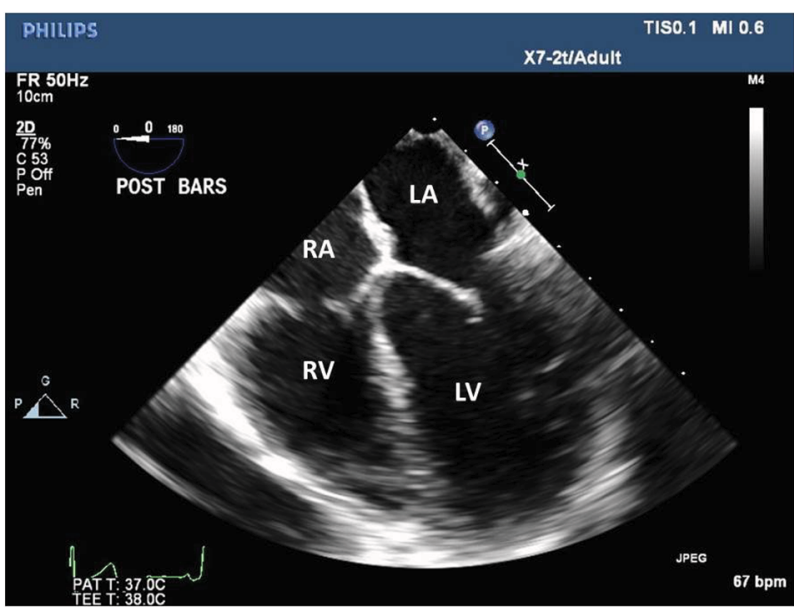

Figure 2 Transesophageal echocardiographic images show preoperative effect $(\mathbf{A})$ of pectus excavatum with compression on the right ventricle due to the inward sternal deformity and relief of the compression following surgical repair (B).

Abbreviations: RV, right ventricle; LV, left ventricle; RA, right atrium; LA, left atrium. 


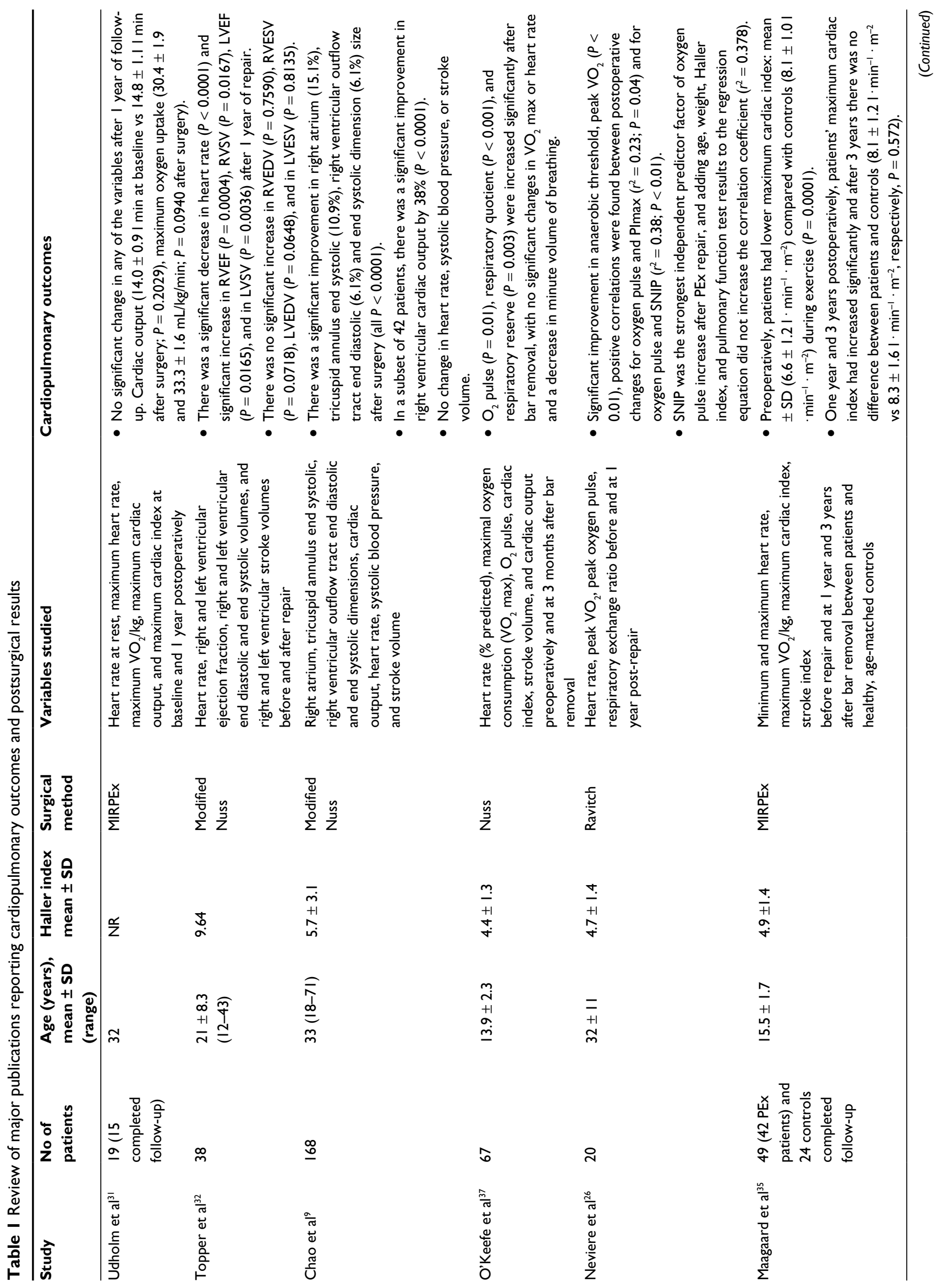




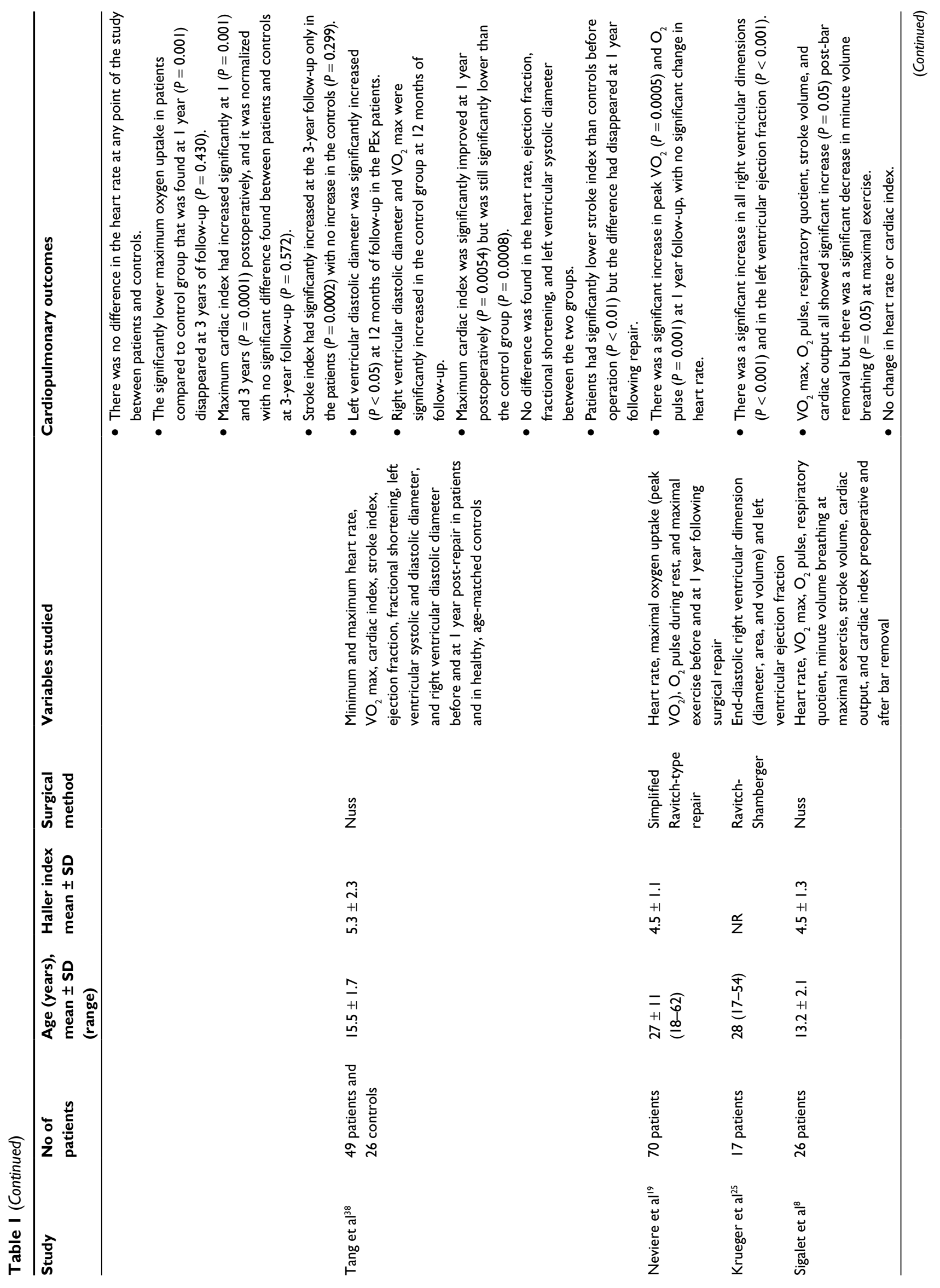




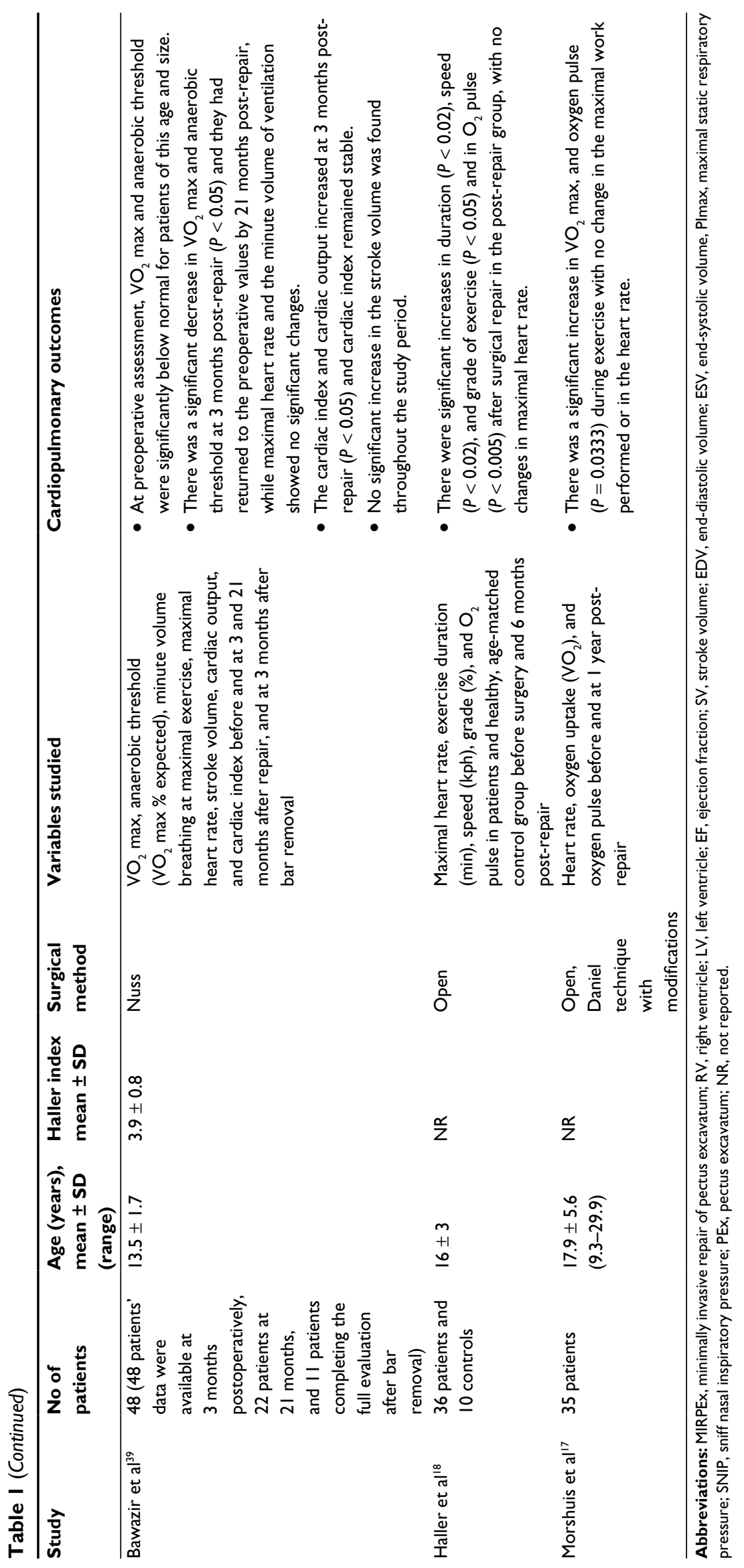


Cardiac output and deconditioning can occur with postoperative inactivity; ${ }^{36}$ therefore, the patient's exercise history can affect the measurements of $\mathrm{VO}_{2}$ and may cause more impact on short-term testing results. ${ }^{34}$

\section{Quality of life and patient satisfaction}

Both the exercise limitations and the cosmetic disfigurement with PEx may cause a decrease in quality of life and alteration of social behavior..$^{37,40-44}$ There has been a greater recognition of the physiologic and psychologic impact of these patients. ${ }^{45}$ Lack of self-confidence, poor body image, avoidance of social activities, and emotional difficulties are noted in PEx patients. Feelings of anxiety, depression, sadness, and frustration are also reported. ${ }^{40}$ The importance of corrective surgery for improvement in psychological distress, quality of life, and exercise tolerance has been documented in the literature. ${ }^{37,41-44}$ The majority of these studies report a mixed population of children and adolescents with few adults; therefore, it is difficult to make broad-based assumptions as to their application to the adult population. ${ }^{46-50}$ Table 2 reviews some of the major publications ${ }^{44,48-50,53,54}$ reporting postsurgical quality of life and symptom outcomes.

Kelly et $\mathrm{al}^{10}$ reported on 264 child patients and 291 parents from multiple centers using a validated Pectus Excavatum Evaluation Questionnaire. Children noted a dramatic improvement in the body image and physical difficulties after surgery. Parents also noticed an improvement in the child's emotional, physical difficulties and social selfconsciousness. ${ }^{10}$ Patient's satisfaction with the chest appearance was found to be very good, with excellent to good results reported in over $95 \%$ of patients at the time of bar removal. ${ }^{51}$

In a 2016 study performed by Lomholt et al, ${ }^{49} 107$ patients and 106 parents completed the generic health-related quality-of-life measure. The Child Health Questionnaire was assessed preoperatively and at 3, 6 months following PEx repair. A control group of 183 school children completed the same measure on one occasion. In the postoperative study, patients and parents reported improved emotional well-being and self-esteem. Additionally, patients at both 3 and 6 months postoperatively reported increased physical and social activities.

There are very few major publications that documented symptoms and quality-of-life improvement after Nuss repair in adult patients. Kragten et $\mathrm{al}^{12}$ reported on symptomatic seniors with PEx. He found that in $45 \%$ of the patients with "serious and sometimes invalidating complaints", symptoms did not start until the fourth or fifth decade of life and were often labeled as "unexplained cardiovascular complaints". All patients that underwent surgery were repaired by the open Ravitch procedure and reported substantial or complete resolution of the symptoms postoperatively. Tikka et $\mathrm{al}^{52}$ used the Brompton's single-step questionnaire (SSQ) to assess the postoperative patient satisfaction and confirmed that Nuss operation had positively impacted the psychological and physical status of their patients along with overall qualityof-life improvement. They reported that their pectus patient information website improved, additionally, their patient's satisfaction and recovery after surgery.

Krasopoulos et $\mathrm{al}^{43}$ proposed the two-step Nuss Questionnaire modified for Adults (NQ-mA) and a SSQ. These questionnaires measured the disease-specific quality-of-life changes after surgery and assessed the effect of surgery on the physical and psychological well-being of postoperative patients. They noted that patients' self-esteem, social functioning, and level of satisfaction were significantly improved following Nuss procedure. Their questionnaire also included the impact of surgical wounds/scars on the overall cosmetic result, consciousness of the presence of metallic bar, the decision to have the operation again, and questions about postoperative pain which may have limited the patient satisfaction after surgery. It was evident from the study that most of the patients were very satisfied with their scars and almost all of them were conscious of the presence of bar, but none of them considered that to be a major inconvenience. Pain was also noted as a concern in the immediate postoperative period; however, it decreased significantly after several weeks. By 4-5 months after surgery, no patient was still requiring analgesics.

Other surgeons have subsequently utilized this modified survey for assessing the patients postoperatively. ${ }^{48,50,53}$ Hoksch et $\mathrm{al}^{50}$ performed a prospective study to evaluate the long-term results of Nuss in adults using NQ-mA and SSQ in a shorter and modified format. Initially, a large adult cohort $(n=129)$ was included, but only19 patients were observed for $>10$ years after surgery. This has been the only study reporting outcomes for an adult population for more than 10 years after surgery. The results obtained initially after surgery were in the follow-up period of 3, 12 and 36 months showed high levels of satisfaction respectively reported at $97.6 \%, 97.2 \%$, and $95.7 \%$. Better or much better quality of life was reported at 3,12 , and 36 months in $88 \%, 89 \%$, and increased to $92.5 \%$, respectively, in the follow-up period. Even after observation for $>10$ years, continued improvement in quality of life was confirmed in $57.9 \%$ of patients. Surgical recommendation for Nuss was given by nearly $95 \%$ of patients. Mild pain 


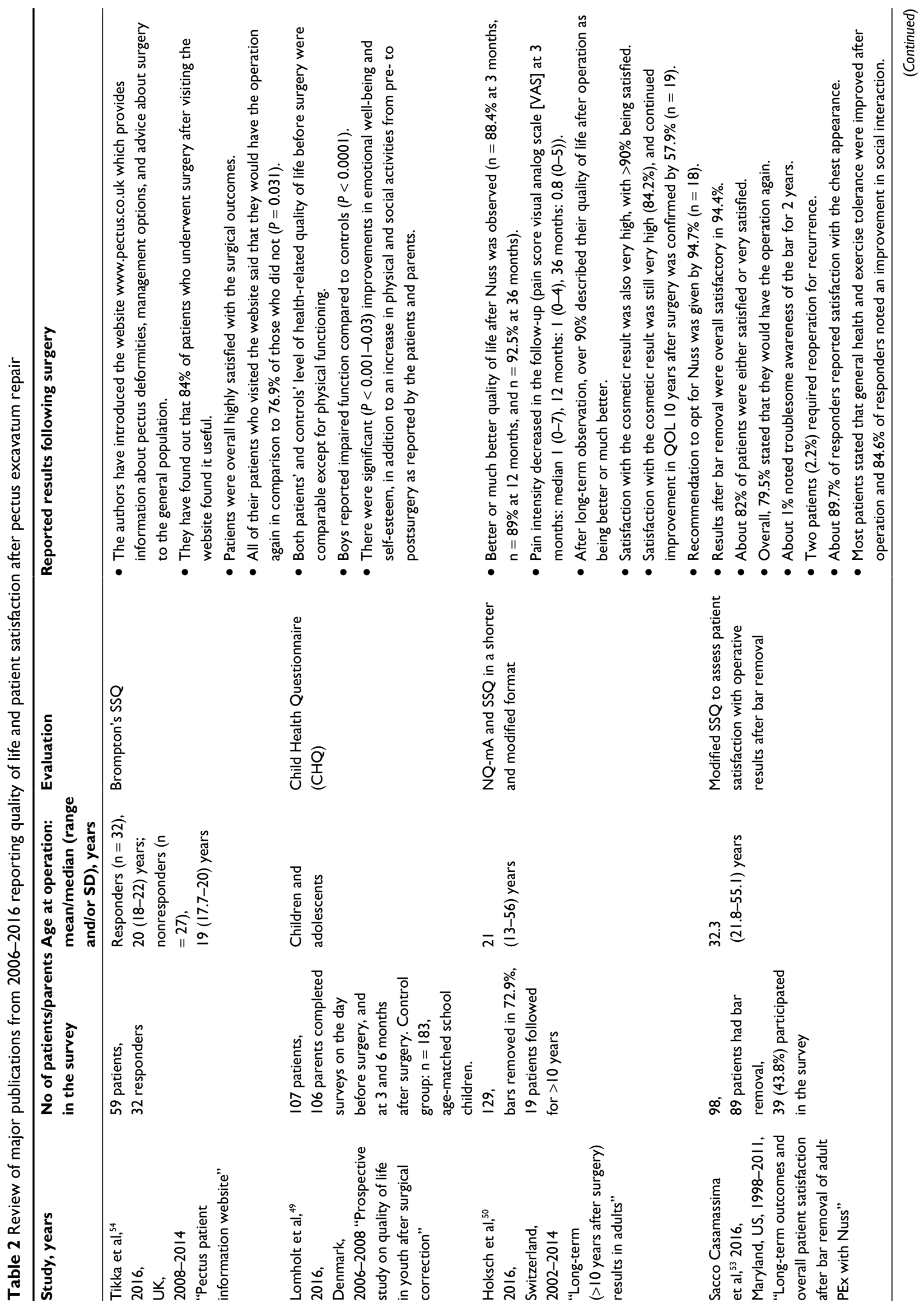




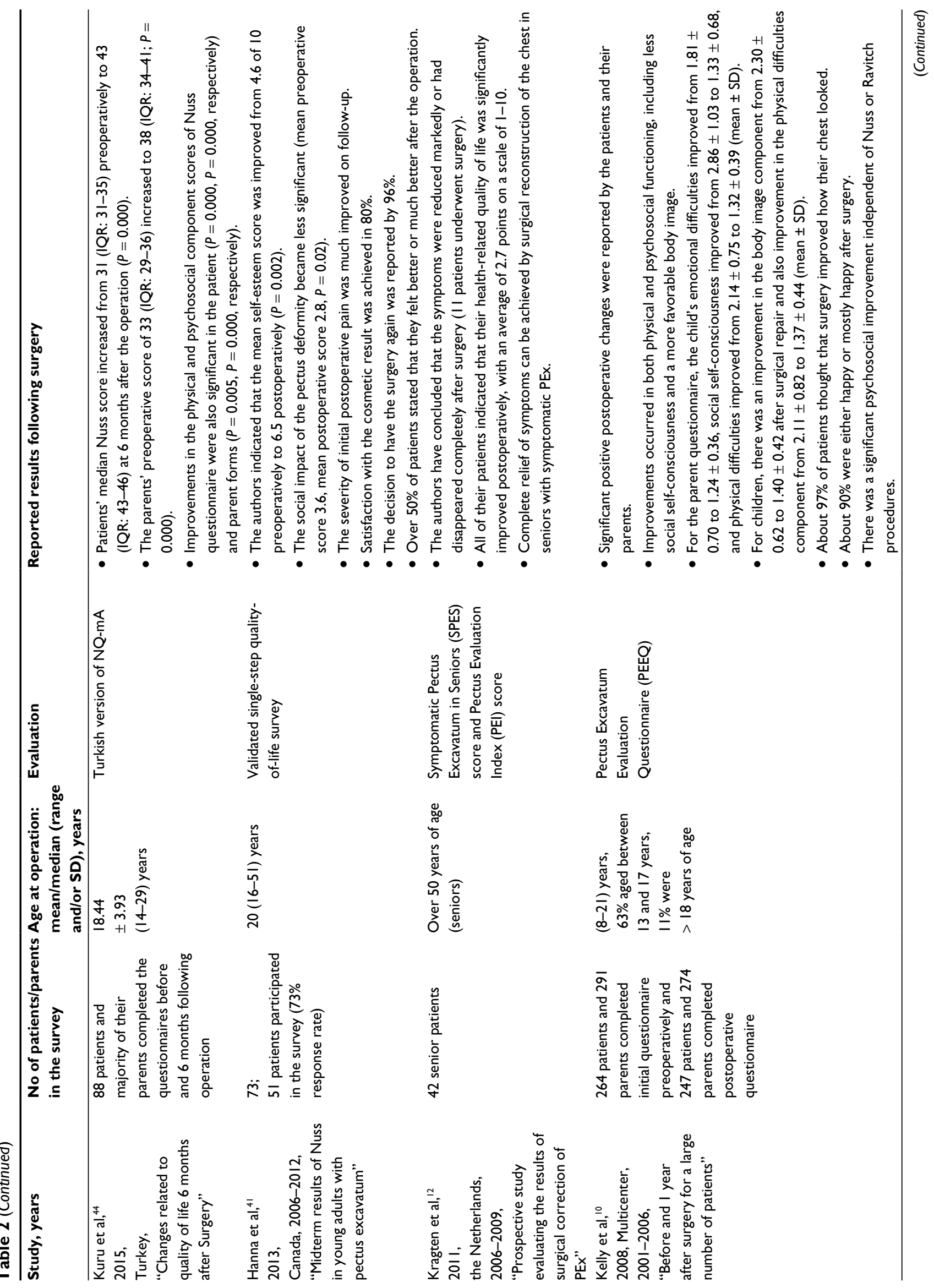




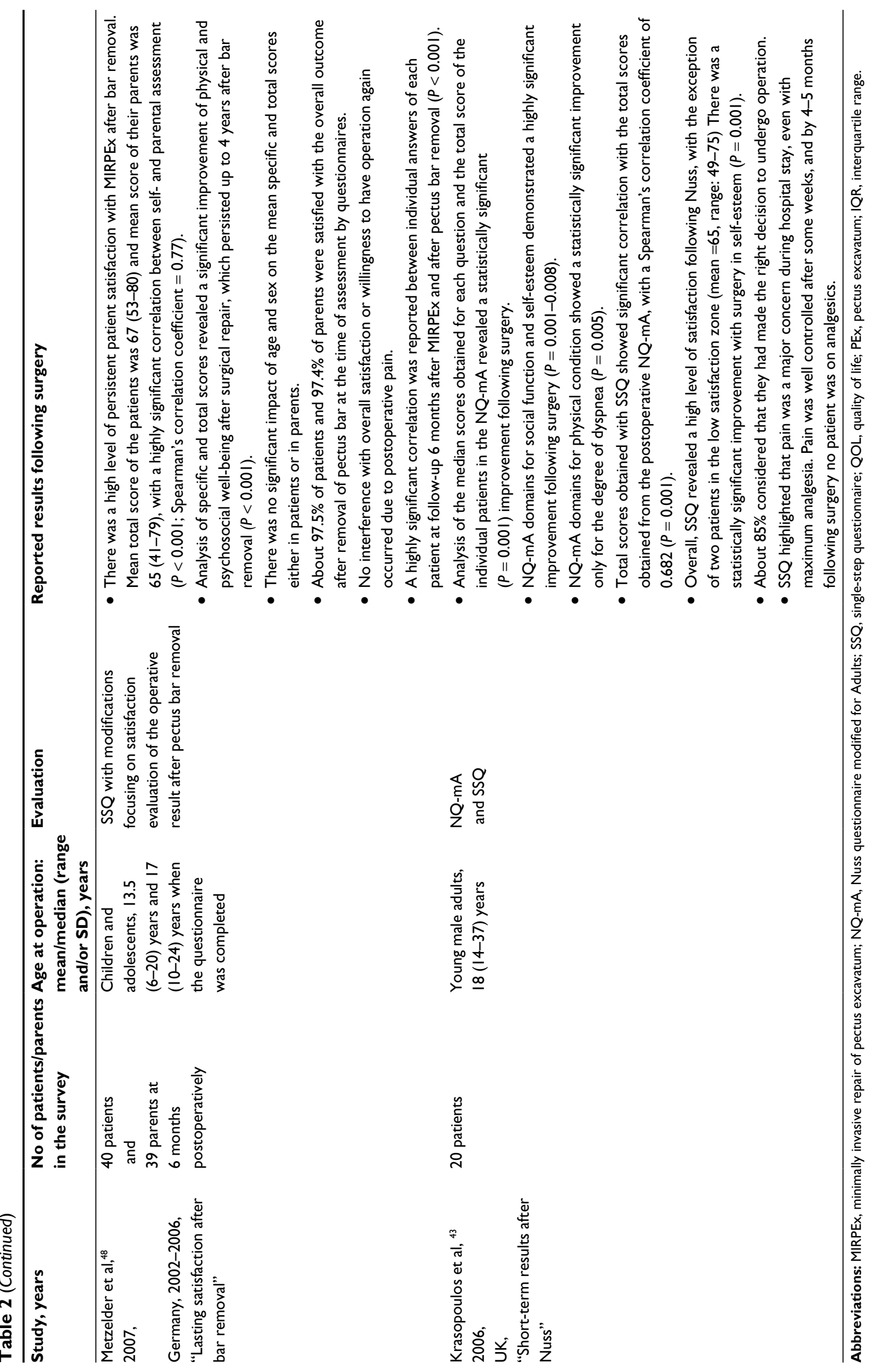


Table 3 Review of some of the recent studies and reported results from 2008-2016 after Nuss procedure for pectus excavatum repair in adults

\begin{tabular}{|c|c|c|c|c|}
\hline $\begin{array}{l}\text { First author, year, } \\
\text { study period }\end{array}$ & $\begin{array}{l}\text { Mean/median, } \\
\text { age (range and/ } \\
\text { or SD), years }\end{array}$ & No of patients & Technique & $\begin{array}{l}\text { Operative time, mean/ } \\
\text { median (range and/or } \\
\text { SD), min }\end{array}$ \\
\hline $\begin{array}{l}\text { Pilegaard, } \\
2016 \\
(2001-2016), \\
\text { Retrospective till } \\
2010 \text { and prospective } \\
\text { afterword }\end{array}$ & $\begin{array}{l}16(7-58) \\
>18 y \\
N=604(35 \%) \\
<18 y \\
N=1109(65 \%)\end{array}$ & 1713 & $\begin{array}{l}\text { MIRPEx } \\
\text { Short bar technique } \\
\text { Unilateral stabilizer close to the hinge point } \\
\text { with sutures to fix the bar on the opposite } \\
\text { side } \\
\text { In the last } 6 \text { years, no additional sutures } \\
\text { were used, and the bar has been placed } \\
\text { asymmetrically on the chest } \\
\text { One bar: } 70.6 \% \\
\text { Two bars: } 28.4 \% \\
\text { Three bars: } 1 \% \\
\text { (in four patients, there was combined } \\
\text { correction of PEx with a cardiac operation) }\end{array}$ & $36(12-270)$ \\
\hline $\begin{array}{l}\text { Jaroszewski et al, }{ }^{24} \\
2016 \\
(2010-2015)\end{array}$ & $\begin{array}{l}23.7 \text { (I8-29), } 40.4 \\
(30-72)\end{array}$ & $\begin{array}{l}26618-29 y \\
n=115 \\
(43.2 \%) \\
30-72 y, n=15 I \\
(56.8 \%)\end{array}$ & $\begin{array}{l}\text { Thoracoscopic MIRPEx } \\
\text { Forced sternal elevation with sternal } \\
\text { bone clamp attached to a bedside Rultract } \\
\text { retractor. } \\
\text { Multiple bars } \\
\text { FiberWire multipoint fixation }\end{array}$ & $\begin{array}{l}\text { I8-29 y cohort, MIRPEx:I I I } \\
(62-178) \text { Hybrid: } 247.5 \\
(138-395) \\
30-72 \text { y cohort, } \\
\text { MIRPEx: } 121(60-224) \\
\text { Hybrid:23I.I(106-390) }\end{array}$ \\
\hline $\begin{array}{l}\text { Pawlak et al, }{ }^{56} 2016 \\
(2002-2012)\end{array}$ & $\begin{array}{l}\text { I8.2 } \pm-5.4(7-49) \\
\text { Groups by age: } \\
\text { A: } \\
\text { I } 2.2 \pm 2.0(7-14) \\
\text { y } \\
\text { B: } \\
\text { I7.2 } \pm 1.6(\mid 5-20) \\
\text { y } \\
\text { C: } \\
25.2 \pm 4.8(2 \mid-49) \\
\text { y }\end{array}$ & $\begin{array}{l}\text { 680, } \\
\text { Groups: } \\
\begin{array}{l}A(n=156), B \\
(n=328), C \\
(n=196)\end{array}\end{array}$ & $\begin{array}{l}\text { Thoracoscopic MIRPEx } \\
\text { Unilateral stabilizers } \\
\text { No of bars Groups: } \\
\text { A, B, C, respectively } \\
\text { One bar: } \\
A=57.6 \% \\
B=50.3 \% \\
C=36.7 \% \\
(P=0.0021) \\
\text { Two bars: } \\
A=41 \% \\
B=48.7 \% \\
C=61.7 \% \\
\text { Three bars: } \\
A=1.2 \% \\
B=0.9 \% \\
C=1.5 \%\end{array}$ & $\begin{array}{l}A, B \text {, and } C \text {, respectively } \\
\text { A: } 50.2 \pm 19.1(25-165) \\
\text { B: } 50.9 \pm 19.8(15-170) \\
C: 55.4 \pm 22(25-200) \\
(P=0.0030)\end{array}$ \\
\hline
\end{tabular}




\begin{tabular}{|c|c|c|c|c|}
\hline $\begin{array}{l}\text { LOS, mean/ } \\
\text { median (range } \\
\text { and/or SD), days }\end{array}$ & Complications, \% & Redo, \% & $\begin{array}{l}\text { Patients } \\
\text { with bars } \\
\text { removed, \% }\end{array}$ & Results \\
\hline $\begin{array}{l}\text { The median LOS } \\
\text { decreased over time } \\
\text { from } 6 \text { (4-29) to } 2 \\
\text { days } \\
\text { Currently, I.6\% } \\
\text { of patients stay } \\
\text { more than } 4 \text { days, } \\
\text { compared with } 46 \% \\
\text { during the early } \\
\text { study period }\end{array}$ & $\begin{array}{l}\text { Bar rotation ( } 2 \mathrm{I} \text { cases }[\mathrm{I} .2 \%] \text { ) and } \\
\text { dislocation ( } 13 \text { cases [0.8\%]) } \\
\text { Deep infection: } 0.9 \% \\
\text { Fractura sterni (one patient): } 0.06 \% \\
\text { *Sternotomy (two cases): } 0.1 \% \\
\text { Pneumonia: } 0.6 \% \\
\text { Pneumothorax: } 1.1 \% \\
\text { Seroma: } 0.4 \% \\
\text { Pleural effusion: } 0.3 \% \\
\text { Bleeding requiring re-operation: } 0.1 \% \\
\text { Bar end dropped into chest cavity: } 0.2 \% \\
\text { Removal of bar before time: } 0.4 \% \\
\text { Stabilizer was removed because of pain: } 1.4 \%\end{array}$ & NR & NR & $\begin{array}{l}\text { No mortality. } \\
\text { Over the study period, there was increase in } \\
\text { the number and decrease in the length of the } \\
\text { bars used. } \\
\text { The duration of postoperative hospitalization } \\
\text { decreased over the study period with the } \\
\text { majority of patients ( }>85 \%) \text { currently being } \\
\text { discharged on the second postoperative day. }\end{array}$ \\
\hline $\begin{array}{l}20 \mid 3-2015 \text { I8-29 } \\
\text { y cohort, MIRPEx: } \\
\text { 3.I (2-6) Hybrid: } 6.5 \\
(6-7) \\
30-72 \text { y cohort, } \\
\text { MIRPEx: } 3.3(2-6) \\
\text { Hybrid: } 5.6(3-11)\end{array}$ & $\begin{array}{l}\text { I8-29 y cohort vs } 30-72 \text {, respectively } \\
\text { Bar rotation: } 1.7 \% \text { vs } 6.6 \% \\
\text { Infection: } 0.9 \% \text { vs } 1.3 \% \\
\text { Pleural effusion (and thoracentesis): } 2.6 \% \\
\text { vs } 6 \% \\
\text { Pneumothorax requiring chest tube: } 0.9 \% \\
\text { vs } 0.7 \% \\
\text { Pulmonary embolism: } 0 \% \text { vs } 1.3 \% \\
\text { Bleeding requiring transfusion: } 0 \% \text { vs } 0.7 \% \\
\text { Reoperation for bleeding: } 1.7 \% \text { vs } 0.7 \% \\
\text { Pneumonia } 0 \% \text { vs } 4 \% \\
\text { Urinary tract infection: } 0 \% \text { vs } 4 \% \\
\text { Urinary retention required catheterization: } \\
7.8 \% \text { vs } 8.6 \% \\
\text { Readmission for pain control: } 0 \% \text { vs } 2 \% \\
\text { One patient with subjective report of } \\
\text { regression in > } 30 \text { group. }\end{array}$ & NR & $19 \%,>30 y$ & $\begin{array}{l}\text { MIRPEx was successfully performed in } 88.7 \% \\
\text { of adults } \geq 30 \text { years and in } 96.5 \% \text { of patients } \\
\text { between } 18 \text { and } 29 \text { years. } \\
\text { Higher percentage of older patients required } \\
\text { osteotomy or cartilage resection ( } 11.3 \% \text { vs } \\
3.5 \%) \text {. } \\
\text { Although greater, the frequency of bar } \\
\text { rotation requiring reoperation was not } \\
\text { significantly increased in the older patients } \\
(P=0.74) \text {. } \\
\text { For complete correction, three bars were } \\
\text { required in }>40 \% \text { of adult patients. } \\
\text { Older patients had a } 65.2 \% \text { increase in right } \\
\text { ventricular output on transesophageal echo } \\
\text { intraoperatively. } \\
\text { No recurrences. }\end{array}$ \\
\hline NR & $\begin{array}{l}A, B \text {, and } C \text { respectively. Pneumothorax: } \\
A=14.7 \% \\
B=27.1 \% \\
C=22.4 \% \\
(P=0.0099) \\
\text { Requiring drainage: } A=26.1 \% \\
B=27 \% \\
C=50 \% \\
\text { Pleural effusion: } A=5.7 \% \\
B=6.4 \% \\
C=11.2 \% \\
(P=0.1436) \\
\text { Requiring chest tube or thoracentesis: } \\
A=77.7 \% B=90.5 \% \\
C=72.7 \% \text { Pleural hematoma: } \\
A=0.6 \% \\
B=0.9 \% \\
C=0.5 \% \\
\text { Fever: } A=1.9 \% \\
B=3.6 \% \\
C=4.6 \% \\
(P=0.3969)\end{array}$ & & NR & $\begin{array}{l}\text { Good cosmetic results reported with the use } \\
\text { of Nuss irrespective of age of the patients. } \\
\text { Good and very good corrective results in } \\
97.7 \% \text { of the patients. } \\
\text { Satisfactory corrective effect: } \\
A=96.8 \% \\
B=98.7 \% \\
C=96.4 \% \\
\text { vs unsatisfactory effect: } \\
A=3.2 \% \\
B=1.2 \% \\
C=3.6 \% \\
(P=0.4563)\end{array}$ \\
\hline
\end{tabular}


Table 3 (Continued)

\begin{tabular}{llll}
\hline $\begin{array}{l}\text { First author, year, } \\
\text { study period }\end{array}$ & $\begin{array}{l}\text { Mean/median, No of patients } \\
\text { age (range and/ } \\
\text { or SD), years }\end{array}$ & $\begin{array}{l}\text { Technique } \\
\text { median (range and/or }\end{array}$ & SD), min \\
\hline
\end{tabular}

Ersen et al, ${ }^{57} 2016$

(2006-2016)
I $6.8(2-45)$ y

Adults: 23.2

( I8-45) y
836

Adults: $\mathrm{n}=236$

$(28.2 \%>18 y)$
Thoracoscopic MIRPEx

Left-sided stabilizer placed and medial

bar secured on right side with

polydioxanone (PDS) sutures around ribs.

2/ > bars:

$15.8 \%$ adults and

$7.5 \%$ of younger patients

$(P=0.068)$

Shorter bars:

median length: II inches (9-14) in adults and

10 inches (7-14) in younger patients
44.4

(25-90)

$\begin{array}{ll}\text { Sacco Casamassima } & 30.9(21.8-55.1) \\ \text { et } a l,,^{53} 2016 & y \\ (1998-2011) & \end{array}$

98 ,

39 patients from 89 who underwent bar removal participated in the survey $(43.8 \%$ response rate)
MIRPEx without thoracoscopy

Bilateral stabilizers

One bar: $89.7 \%$;

Two bars: $10.2 \%$
$62.9 \pm 24.9$ 


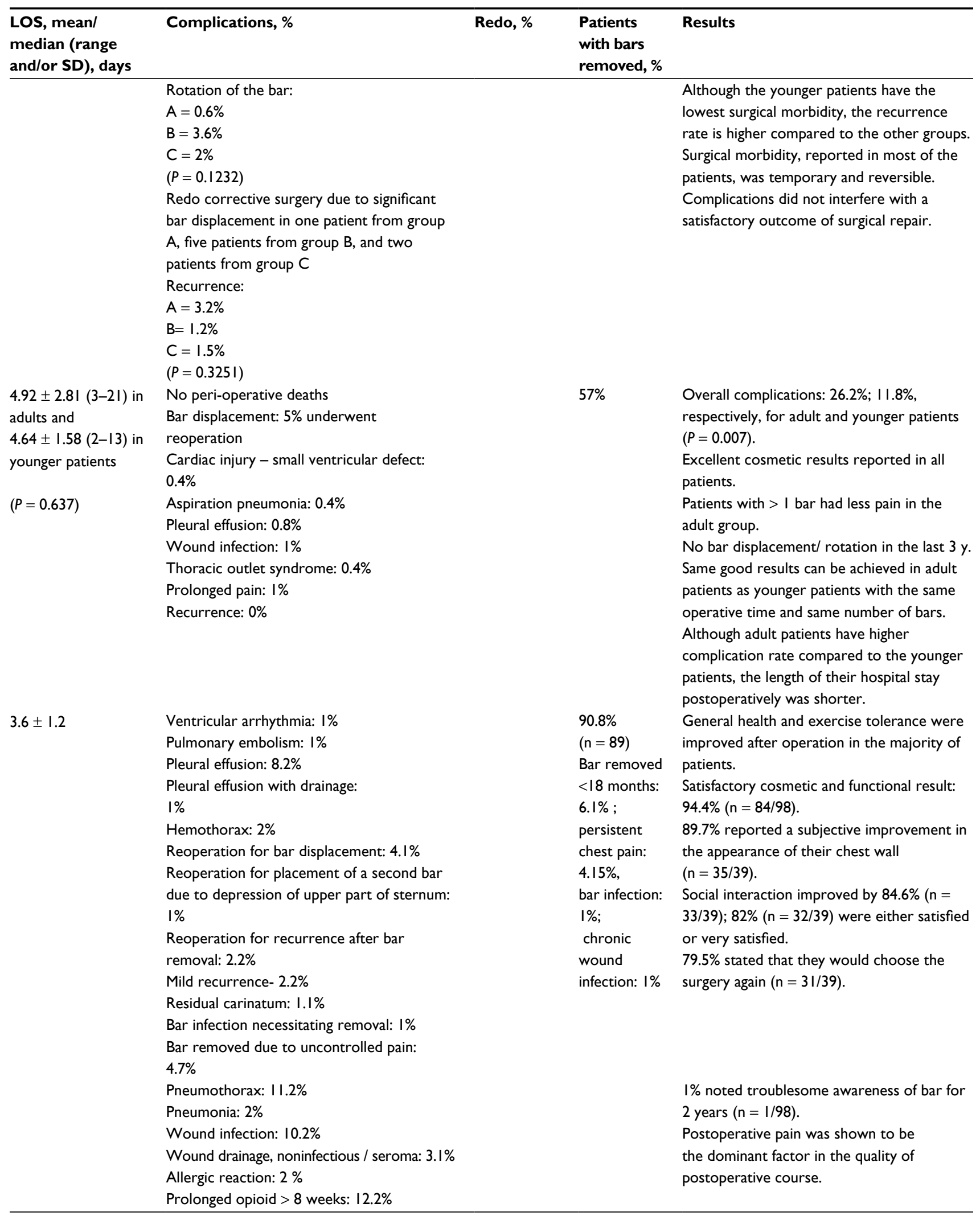


Table 3 (Continued)

\begin{tabular}{|c|c|c|c|}
\hline $\begin{array}{l}\text { First author, year, } \\
\text { study period }\end{array}$ & $\begin{array}{l}\text { Mean/median, } \\
\text { age (range and/ } \\
\text { or SD), years }\end{array}$ & No of patients & Technique \\
\hline $\begin{array}{l}\text { Fibla et al, }{ }^{58} 2016 \\
(2001-2010)\end{array}$ & $21.2(10-47)$ & $\begin{array}{l}\text { I49, } \\
\text { the surgery could } \\
\text { not be concluded } \\
\text { in two patients due } \\
\text { to the inability to } \\
\text { elevate the sternum } \\
\text { ( } 147 \text { used for } \\
\text { calculations in some } \\
\text { instances) }\end{array}$ & $\begin{array}{l}\text { Multi center MIRPEx most with } \\
\text { thoracoscopy, few Ravitch included } \\
\text { Stabilization not reported } \\
\text { One bar: } 94.6 \% \text {; } \\
\text { Two bars: } 5.4 \%\end{array}$ \\
\hline Park et al, ${ }^{59} 2015$ & 10.3 & 1816 & MIRPEx thoracoscopy/pectoscopy \\
\hline$(1999-2012)$ & $\begin{array}{l}\text { (16 months-53 y) } \\
\text { Adults >15 y: } \\
21.4 \%\end{array}$ & $\begin{array}{l}\text { Groups according } \\
\text { to the bar fixation } \\
\text { method: }\end{array}$ & $\begin{array}{l}\text { Series with different bar fixation techniques } \\
\text { Stabilizers } \\
\text { Multipoint pericostal suture fixation } \\
\text { Claw fixator }\end{array}$ \\
\hline & & $\begin{array}{l}\text { STB: } n=180 \\
\text { MPF: } n=760 \\
\text { CFT: } n=699 \\
\text { CFT }+ \text { HP }: n=177\end{array}$ & Hinge plate \\
\hline
\end{tabular}

Operative time, mean/ median (range and/or SD), min

NR NR

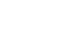




\begin{tabular}{|c|c|c|c|c|}
\hline $\begin{array}{l}\text { LOS, mean/ } \\
\text { median (range } \\
\text { and/or SD), days }\end{array}$ & Complications, \% & Redo, \% & $\begin{array}{l}\text { Patients } \\
\text { with bars } \\
\text { removed, \% }\end{array}$ & Results \\
\hline $\mathrm{NR}$ & $\begin{array}{l}\text { Bar displacement: } 5.4 \% \text { (with } 3.4 \% \text { requiring } \\
\text { reoperation) } \\
\text { Seroma in surgical wounds: I } 1.6 \% \\
\text { Breakage of the absorbable stabilizer: } 2 \% \\
\text { Pneumothorax requiring drainage: } 2 \% \\
\text { Hemothorax: } 1.4 \% \text { (one patient required } \\
\text { reoperation) } \\
\text { Wound infection: } 2.7 \% \\
\text { Pericarditis: } 0.7 \% \\
\text { Pericardial blockage and respiratory failure } \\
\text { necessitating emergency bar removal: } 0.7 \% \\
\text { All patients reported postoperative pain with } \\
2 \% \text { went bar removal due to intense pain }\end{array}$ & & $\begin{array}{l}49 \% \\
\text { Difficult: } \\
7 \%\end{array}$ & $\begin{array}{l}\text { Initial results: } \\
\text { Excellent/good: } 93.2 \% \\
\text { Mild: } 4.1 \% \\
\text { Bad: } 2.7 \% \\
\text { After a } 1.6 \text {-year follow-up period, good } \\
\text { results persisted in } 98.7 \% \\
\text { Result was not satisfactory in I.4\% } \\
\text { reoperated using Ravitch. } \\
\text { Complications: } 30.6 \% \\
\text { In } 32 \text { and } 37 \text { year old patients, surgery was } \\
\text { converted to Ravitch due to impossibility of } \\
\text { raising the sternum. }\end{array}$ \\
\hline NR & $\begin{array}{l}\text { STB vs MPF vs CFT + HP, respectively } \\
\text { Total complication: } \\
\text { STB: } 20 \% \\
\text { MPF: I I.2\% CFT + HP: } 7.3 \% \\
\text { Early bar displacement: } \\
\text { STB: } 3.33 \% \\
\text { MPF: } 0.56 \% \\
\text { CFT + HP: } 0 \% \\
\text { Reoperation: } \\
\text { STB: } 5 \% \\
\text { MPF: I.57\% } \\
\text { CFT + HP: } 3.38 \% \\
\text { Pneumothorax: STB: I0.3\% MPF: } 2.6 \% \text { CFT } \\
\text { + HP: } 0.5 \% \\
\text { Pleural effusion: STB: } 3.3 \% \text { MPF: } 2.5 \% \text { CFT } \\
+ \text { HP: } 2.9 \% \\
\text { Pericardial effusion: STB: I.6\% MPF: } 0.3 \% \\
\text { CFT + HP: } 0.2 \% \\
\text { Wound seroma: STB: } 5.4 \% \text { MPF: } 3.7 \% \text { CFT } \\
+ \text { HP: I.5\% } \\
\text { There were four cases (0.32\%) of reoperation } \\
\text { because of recurrence after bar removal }\end{array}$ & & $\begin{array}{l}\text { Pectus } \\
\text { bars were } \\
\text { removed } \\
\text { from I23। } \\
\text { patients } \\
(67.7 \%)\end{array}$ & $\begin{array}{l}\text { Total complication rates lower in CFT }+\mathrm{HP} \\
\text { (I4.I\%) than STB group }(22.7 \%),(P<0.0 \mathrm{I}) \text {. } \\
\text { Repair has been durable in more than } 99 \% \\
\text { who had bar removal during follow-up } \\
\text { period of } 10 \mathrm{y} \text {. } \\
\text { Pericostal suture fixation is vital for securing } \\
\text { the stabilizer to function appropriately. } \\
\text { There was a shift from the use of stabilizer } \\
\text { to use of pericostal sutures to fix the bar } \\
\text { (multipoint fixation). } \\
\text { Suture-less claw fixator was equally as } \\
\text { effective as MPF. }\end{array}$ \\
\hline $\begin{array}{c}5.2 \pm 2.9 \\
(4-36)\end{array}$ & $\begin{array}{l}\text { One postoperative death due to right atrial } \\
\text { injury: } 0.2 \% \\
\text { Bar displacement: } 1.7 \% \\
\text { Bar displacement requiring reoperation: } \\
0.6 \% \\
\text { Pericardial perforation: } 1.3 \% \\
\text { Intercostal tear: } 0.8 \% \\
\text { Wound infection: } 0.5 \% \\
\text { Pneumothorax: } 2.8 \% \\
\text { Pneumonia: } 0.6 \% \\
\text { Pleural effusion: } \\
0.8 \% \\
\text { Hemothorax: } 0.2 \%\end{array}$ & & $47.6 \%$ & $\begin{array}{l}\text { Outcomes were excellent: } 78.9 \% \text {; } \\
\text { good: } 16.4 \% \text {; } \\
\text { fair: } 4.4 \% \text {; } \\
\text { poor: } 0.4 \% \text {. } \\
\text { Good quality rate: } 95.3 \% \text {. } \\
\text { Adult patients significantly required more } \\
\text { pectus bars compared to the youth. } \\
\text { Within the average } 31.1 \text { months follow-up, } \\
\text { no significant recurrences } \\
\text { were reported. } \\
\text { Complication rate: } \\
9.7 \% \text {. }\end{array}$ \\
\hline NR & $\begin{array}{l}\text { Complication rate: } 7.5 \% \\
\text { Wound hematoma: } 1.25 \% \\
\text { Wound dehiscence: } 1.25 \% \\
\text { Wound infections: } 2.5 \% \\
\text { Pleural effusions: } 2.5 \% \\
\text { Bar displacement: } 0 \%\end{array}$ & & NR & $\begin{array}{l}\text { Over a 4-month follow-up period, there was } \\
\text { no reported movement in the upper and } \\
\text { lower bars, and there were no cases of bar } \\
\text { displacement or reoperation. } \\
\text { No suture fixations or invasive devices were } \\
\text { required in the bridge technique, making it } \\
\text { applicable to use. }\end{array}$ \\
\hline
\end{tabular}


Table 3 (Continued)

\begin{tabular}{|c|c|c|c|c|}
\hline $\begin{array}{l}\text { First author, year, } \\
\text { study period }\end{array}$ & $\begin{array}{l}\text { Mean/median, } \\
\text { age (range and/ } \\
\text { or SD), years }\end{array}$ & No of patients & Technique & $\begin{array}{l}\text { Operative time, mean/ } \\
\text { median (range and/or } \\
\text { SD), min }\end{array}$ \\
\hline $\begin{array}{l}\text { Hanna et al, }{ }^{41} 2013 \\
(2006-2012)\end{array}$ & $\begin{array}{l}20(16-51) y \\
16 \% \text { between } 16 \\
\text { and } 18 y\end{array}$ & $\begin{array}{l}73 \\
51 \text { patients agreed } \\
\text { to participate } \\
\text { in a quality-of- } \\
\text { life survey ( } 73 \% \\
\text { response rate) }\end{array}$ & $\begin{array}{l}\text { MIRPEx with thoracoscopy. } \\
\text { Bilateral stabilizers, } \\
\text { two wires to adjacent ribs } \\
\text { One bar: } 81 \% \text {, } \\
\text { Two bars: } 19 \%\end{array}$ & NR \\
\hline
\end{tabular}

\begin{tabular}{|c|c|c|c|c|}
\hline Rokitansky and & $17.7 \pm 7$ & 262 & MEMIPR : MMIPR + partial sternotomy & NR \\
\hline Stanek, ${ }^{62} 2013$ & Majority of & MMIPR: & $(23 \%)$ & \\
\hline \multirow[t]{10}{*}{$(2006-2013)$} & patients between & $n=121$ & Stabilizer wing (PSI by Hofer Medical, & \\
\hline & 14 and $20 y$ & MEMIPR: & Austria) & \\
\hline & MMIPR: & $n=14 \mid$ & Slit-rib chondrotomy under thoracoscopic & \\
\hline & $15.2 \pm 5$ & & guidance $(48 \%)$ & \\
\hline & MEMIPR: & & rib resection (5\%), and rib osteotomy & \\
\hline & $22.5 \pm 8$ & & MMIPR: & \\
\hline & & & ; Symmetrical PEx: 74\% & \\
\hline & & & Two bars: $13.2 \%$ & \\
\hline & & & MEMIPR: symmetrical PEx: $57.4 \%$, carinatum & \\
\hline & & & /excavatum: $4.9 \%$, two bars: $58.1 \%$ & \\
\hline Olbrecht et al, ${ }^{63}$ & $23(18-30)$ & 18-30 y: & MIRPEx without routine use of & $82(65.5-103.5)$ \\
\hline 2008 & & $\mathrm{n}=107$ & thoracoscopic visualization & \\
\hline$(1997-2006)$ & & (52 bars removed) & Lateral stabilizers & \\
\hline \multirow[t]{4}{*}{ Values: median (IQR) } & & 6-14 y: & Sternal wire to anchor bar to crossing ribs & \\
\hline & & $n=137$ & bilaterally, & \\
\hline & & (80 bars removed) & One bar: $94.2 \%$ Two bars: $5.8 \%$ & \\
\hline & & & $(P=0.03)$ & \\
\hline
\end{tabular}




\begin{tabular}{|c|c|c|c|c|}
\hline $\begin{array}{l}\text { LOS, mean/ } \\
\text { median (range } \\
\text { and/or SD), days }\end{array}$ & Complications, \% & Redo, \% & $\begin{array}{l}\text { Patients } \\
\text { with bars } \\
\text { removed, \% }\end{array}$ & Results \\
\hline $5(3-9)$ & $\begin{array}{l}\text { Bar displacement: } 2.7 \% \text { (required } \\
\text { reoperation) } \\
\text { Self-resolving pneumothorax: } 4.1 \% \\
\text { Poor cosmesis: } 2.7 \% \text { (required revision for } \\
\text { bar positioning) } \\
\text { Bar infection: } 1.4 \% \\
\text { Bruising: } 1.4 \% \\
\text { lleus: } 1.4 \% \\
\text { Pericarditis: } 1.4 \% \\
\text { Immediate postoperative pain was severe in } \\
51 \% \text { and very severe in } 39 \%\end{array}$ & & $57 \%$ & $\begin{array}{l}\text { Mean self-esteem score significantly } \\
\text { improved after surgery from } 4.6 \text { to } 6.5 \text { out } \\
\text { of } 10 \text { postoperatively } \\
(P=0.002) \text {. } \\
\text { Mean of social impact of pectus deformity } \\
\text { became less significant ( } 3.6 \text { to } 2.8) \text {, } \\
(P=0.02) \\
\text { Overall morbidity rate: } 15.1 \% \text {. } \\
\text { No incidents of bar displacement were } \\
\text { reported after using the stainless steel wires } \\
\text { instead of polypropylene. } \\
\text { Severity of initial postoperative pain was } \\
\text { improved on follow-up with } 84 \% \text { reporting } \\
\text { no pain or occasional pain with no need for } \\
\text { analgesia. } \\
80 \% \text { were satisfied with the cosmetic result } \\
\text { and } 96 \% \text { would choose to undergo surgical } \\
\text { repair again. } \\
\text { No documented recurrence of PEx after bar } \\
\text { removal. }\end{array}$ \\
\hline NR & $\begin{array}{l}\text { MMIPR, MEMIPR, respectively } \\
\text { No bar displacement, only minimal } \\
\text { bar movement: } \\
\text { MMIPR: } 4.9 \% \\
\text { MEMIPR: } 1.6 \% \\
\text { Stabilizer dislocation: MMIPR: } 2.3 \% \\
\text { MEMIPR: } 0 \% \\
\text { Subcutaneous infection: MMIPR: } 0.7 \% \\
\text { MEMIPR: } 0.8 \% \\
\text { Pneumothorax: } \\
\text { MMIPR: } 1.4 \% \\
\text { MEMIPR: } 2.4 \% \\
\text { Pleural effusion (needs drain): } \\
\text { MMIPR: } 2.1 \% \\
\text { MEMIPR: } 1.7 \% \\
\text { Subcutaneoushematoma: MMIPR: I.7\% } \\
\text { MEMIPR: } 4.1 \% \\
\text { Tissue necrosis: } \\
\text { MMIPR: } 2.5 \% \\
\text { MEMIPR: } 0 \% \\
\text { Recurrence occurred in } 0.9 \% \text { of patients } \\
\text { who underwent bar removal, with one } \\
\text { patient undergoing early bar removal and } \\
\text { required correction of a recurrent funnel } \\
\text { chest }\end{array}$ & & $\begin{array}{l}\text { I03 patients } \\
\text { with a mean } \\
\text { of } 3.4 \mathrm{y} \\
(1.4-6.5)\end{array}$ & $\begin{array}{l}\text { MMIPR and MEMIPR yielded very satisfactory } \\
\text { results, especially in older patients with } \\
\text { severe deformities and recurrence. } \\
\text { Simple MIPR did not yield the desired } \\
\text { results in elderly patients with stiff thorax, } \\
\text { curved sternum, severe asymmetrical forms, } \\
\text { and a mixed pigeon/funnel chest in whom } \\
\text { the modification procedures (MMIPR and } \\
\text { MEMIPR) resulted in very satisfactory } \\
\text { outcomes. } \\
\text { About } 95 \% \text { of the patients were satisfied } \\
\text { with the cosmetic outcome after bar } \\
\text { removal. } \\
\text { None of the } 262 \text { patients who underwent } \\
\text { MMIPR or MEMIPR required re- } \\
\text { thoracotomy. }\end{array}$ \\
\hline $3(3-4)$ & $\begin{array}{l}\text { Bar displacement requiring operation: } 7.7 \% \\
\text { Pneumothorax: requiring tube: } 3.9 \% \\
\text { Pleural effusion: } 3.9 \% \\
\text { Pneumonia: } 3.9 \% \\
\text { Superficial surgical site infection: } 21.6 \% \\
\text { (requiring surgical revision: } 5.9 \% \text { Requiring } \\
\text { early bar removal: } 3.9 \% \text { ) }\end{array}$ & & $\begin{array}{l}48.6 \%>18 y \\
58.4 \% \\
6-14 y\end{array}$ & $\begin{array}{l}\text { No patient required open procedure and } \\
\text { two patients required sternal osteotomy. } \\
\text { Operative time shorter and postoperative } \\
\text { complications were similar to Ravitch. } \\
\text { Long-term complication rates were equal } \\
\text { between adult and pediatric cohort. } \\
\text { Adults often require more than one bar for } \\
\text { correction. }\end{array}$ \\
\hline
\end{tabular}


Table 3 (Continued)

\begin{tabular}{llll}
\hline $\begin{array}{l}\text { First author, year, } \\
\text { study period }\end{array}$ & $\begin{array}{l}\text { Mean/median, No of patients } \\
\text { age (range and/ }\end{array}$ & $\begin{array}{l}\text { Technique } \\
\text { or SD), years }\end{array}$ & Operative time, mean/ \\
\hline
\end{tabular}

$\begin{array}{llll}\begin{array}{l}\text { Cheng et al, }{ }^{30} 2008 \\ \text { (2005-2007), }\end{array} & 24.5 & 96 & \text { MIRPEx with bilateral thoracoscopy } \\ \text { prospective } & (I 8-42) & \text { Young adults: } & \text { Two bars: 22.9\%, } \\ & & (I 8-25) y, & \text { Wire/suture bar fixation at right hinge point } \\ & n=63 & \text { and distal bar ends to rib } \\ & \text { Older adults: } \\ & \\ & & \\ & & \\ & & \\ & & \end{array}$

$80(50-185)$

Mean time for first repair with one bar: 65 (50-100); redo correction with two bars: 120 (100-185)

Note: *Both sternotomies were done in re-do cases: one was due to inferior vena cava bleeding and one was due to right ventricle tear caused by an adhesion, from the primary Nuss correction.

Abbreviations: NR, not reported; SD, standard deviation; LOS, length of stay; y, year; MIRPEx, minimally invasive repair of pectus excavatum; STB, stabilizer; MPF, multipoint pericostal fixation; CFT, claw fixator; HP, hinge plate; MIPR, minimally invasive pectus repair; MMIPR, modified minimally invasive pectus repair; MEMIPR, modified extended minimally invasive pectus repair; PEx, pectus excavatum; PC, pectus carinatum; QOL, quality of life; IQR, interquartile range; PSI, Pectus Security Implant.

occurring during specific bodily movements was reported in $31.6 \%$, and $63.2 \%$ of patients had no pain.

Sacco Casamassima et $\mathrm{al}^{53}$ in 2016 reported long-term results of adults using modified SSQ. Satisfaction with the chest wall appearance was reported in $89 \%$ out of $43.8 \%$ of responders. Improvement in social interaction was reported by $84 \%$ of responders. About $94 \%$ of patients obtained overall satisfaction with the results post-bar removal. They also highlighted that the dissatisfaction observed by some patients was due to severe postoperative chest pain (that necessitates more aggressive analgesic regimen) and surgical scars. Willingness to have the operation again was reported by $79 \%$ of responders. Generalized conclusions cannot be drawn from this study as it is limited by small sample size. There is a compelling need for a large number of similar studies commenting on the long-term results in adults to identify the benefits of surgery in this group.

Hanna et $\mathrm{al}^{41}$ studied the midterm results in young adults who underwent Nuss repair and used the single-step quality-of-life survey for evaluation. With a $73 \%$ response rate, they noticed an improvement in both self-esteem and social life. Satisfaction with the cosmetic result was achieved in $80 \%$ and recommendation for the surgery was given by $96 \%$ of their patients. About $92 \%$ reported subjective improvement in the chest wall appearance. As stated by other authors, in-hospital pain despite aggressive analgesic usage was a major concern in the immediate postoperative 


\begin{tabular}{|c|c|c|c|c|}
\hline $\begin{array}{l}\text { LOS, mean/ } \\
\text { median (range } \\
\text { and/or SD), days }\end{array}$ & Complications, \% & Redo, \% & $\begin{array}{l}\text { Patients } \\
\text { with bars } \\
\text { removed, \% }\end{array}$ & Results \\
\hline & $\begin{array}{l}\text { Upper sternal depression requiring } \\
\text { reoperation for second bar: } 3.9 \% \\
\text { Development of pectus carinatum requiring } \\
\text { repair: } 1.9 \% \\
\text { Prolong pain: } 47.1 \% \\
\text { Only two patients ( } 3.9 \%) \text { experienced a } \\
\text { recurrence after bar removal, and neither } \\
\text { had required operative repair }\end{array}$ & & & $\begin{array}{l}\text { Predictors of surgical revision after repair } \\
\text { were bar displacement }(P<0.001) \text { and early } \\
\text { bar removal }(P<0.001) \text {. } \\
\text { Patients did not differ with respect to } \\
\text { postoperative complications, including } \\
\text { recurrence rates, bar displacement, upper } \\
\text { sternal depression, or revision surgery. }\end{array}$ \\
\hline $7.2(5-13)$ & $\begin{array}{l}\text { Number of young adult vs older adult } \\
\text { Bar displacement: } 2 \text { vs } 0 \text { (both required } \\
\text { surgical revision) } \\
\text { Pneumothorax: } 0 \text { vs I } \\
\text { Pleural effusion requiring thoracentesis: I } \\
\text { vs } 0 \\
\text { Pericardial effusion with pericardio-centesis: } \\
0 \text { vs I }\end{array}$ & & $7 \%$ & $\begin{array}{l}\text { About } 91.6 \% \text { of patients were satisfied with } \\
\text { their surgical correction } \\
\text { Satisfaction results were reported as follow } \\
\text { in young and old adults, respectively: } \\
\text { Excellent: } 75.3 \%, 69.7 \% \\
\text { Good: } 17.4 \%, 21.2 \% \\
\text { Fair: } 6.3 \%, 6.1 \% \\
\text { Failed: } 1.6 \%, 3 \% \\
\text { Bilateral thoracoscopy facilitated mediastinal } \\
\text { dissection, particularly in patients who had } \\
\text { previous pectus/thoracic procedure or } \\
\text { double-bar insertion. } \\
\text { Patients with fair or failed results had more } \\
\text { complicated deformities, including } \\
\text { PEx combined with PC, long area deformity, } \\
\text { severe bony rigidity, residual deformity, or } \\
\text { partial recurrence after repair. } \\
\text { Longer operation time and higher } \\
\text { complication rate were reported in } \\
\text { double-bar repair compared to single-bar } \\
\text { procedures. } \\
\text { Single bar ( } \mathrm{n}=74 \text { ): } 3 \% \text {; } \\
\text { double bar } \\
\text { ( } \mathrm{n}=22 \text { ): I } 3.6 \% \text {. }\end{array}$ \\
\hline
\end{tabular}

period; however, in the follow-up it was significantly decreased, with almost all patients reporting minimal or no pain.

Most of the data available suggest that patients who had undergone Nuss showed an overall satisfaction with the cosmetic result, had a significant improvement in self-image, and felt that the surgery had a positive impact on their ability to exercise and well-being.

\section{Surgical approaches and outcomes}

The Nuss procedure or "MIRPEx" has become the standard of care for PEx repair in children and adolescents. ${ }^{64}$ There is an ongoing discussion in the literature regarding the success of this surgery in adults with PEx. Initial reports of Nuss procedure in adults were criticized due to higher complication rates vs the open Ravitch technique with most being related to bar migration, postoperative pain, and recurrences. ${ }^{65-67}$ The recommendations of some surgeons were to limit the procedure to pediatrics and adolescents; however, their publications have been replaced with numerous series of successful repairs using a modified MIRPEx approach. ${ }^{24,68,69}$ Table 3 reviews publications reporting on 70 or more adults repaired using an MIRPEx procedure since 2008. The majority of authors considered patients aged 18 years and older as adults. ${ }^{28,51,57,63,70,71}$ Several papers have stratified their results to differentiate younger vs older patients. ${ }^{24,30}$ There is evidence that older patients are more difficult to treat and the risk of complications may be greater. ${ }^{14}$ Despite this, excellent results are achieved with an MIRPEx approach even in older adult pat ients $^{15,24,28-30,43,63,68,69,71-73}$ (Figure 3A-D). 
Since the introduction of the original Nuss technique for children in $1998,{ }^{64}$ several changes have been made in the surgical technique and methods of bar stabilization which have improved the success of the procedure in adult patients. ${ }^{24,28,72,74-78}$ Important modifications include the use of forced sternal elevation, ${ }^{78}$ multiple support bars, ${ }^{24,77}$ and improved fixation methods to secure the bars and prevent rotation. ${ }^{24,28,72,74-78}$ These technical refinements enabled successful MIRPEx repair of older patients and are reviewed in Table 4.

The use of forced sternal elevation may help reduce the force required to insert and rotate bars (Figure 4). This may lessen, but not eliminate, lateral stripping of the intercostal muscles of the more rigid chest wall. ${ }^{69,78,79,81,82,84}$ Several techniques have been proposed for the forceful elevation of sternum. Park et al ${ }^{79}$ reported his Crane technique and discussed the benefits of its use in adult patients with heavier chests and severely asymmetric deformities including prevention of intercostal muscle tear and bar displacement. Similar variations of this technique have been reported by others with similar beneficial results. ${ }^{69,78,81,82,84}$ A more simplified aspect of handheld retractors can also be utilized, depending on the severity and rigidity of the defect. ${ }^{80,83}$

Multiple bars may balance the increased pressure of the chest wall and in older patients, the use of two or more bars is frequently reported. ${ }^{24,70,77,90}$ The risk of bar rotation and malposition may also be decreased by distributing the pressure of a more rigid chest wall. ${ }^{70,77,90}$ Pilegaard $^{68}$ reported that $70 \%$ of his patients over 30 years of age required two or more bars. In our own practice, two or more bars were utilized in $99 \%$ of patients over age 18 years, ${ }^{24}$ with $40 \%$ of patients over 30 years receiving three bars to achieve complete repair. Others have reported decreased risk of bar migration and the need of reoperation when multiple bars were utilized. ${ }^{70,77,90} \mathrm{In}$ a study of PEx repair in 44 late adolescent and adult patients, $11.5 \%$ of those with single-bar repairs required reoperation for incomplete correction or bar rotation compared with $0 \%$ who had a double-bar repair. Double bar also decreases the postoperative pain as described by Nagaso et al. ${ }^{89}$ The risk of bar rotation may be lowered by the use of shorter bars as reported by several surgeons. ${ }^{93,94}$ In a publication reporting
A

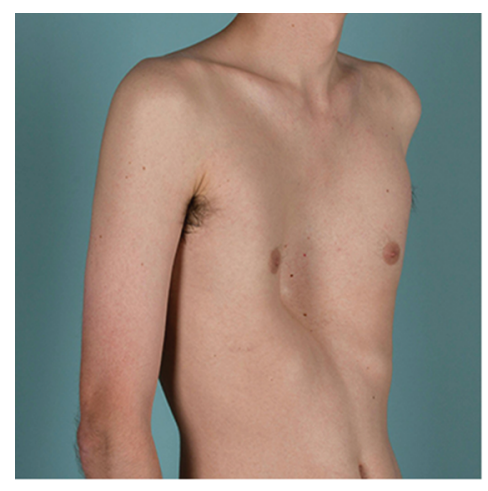

C

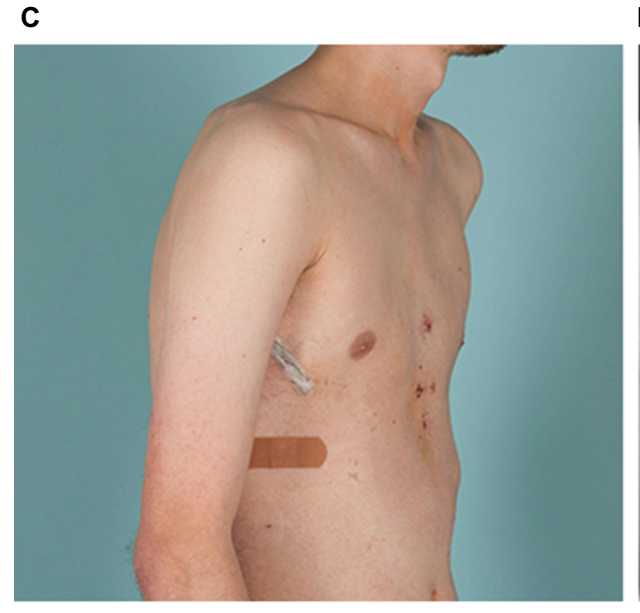

\section{B}

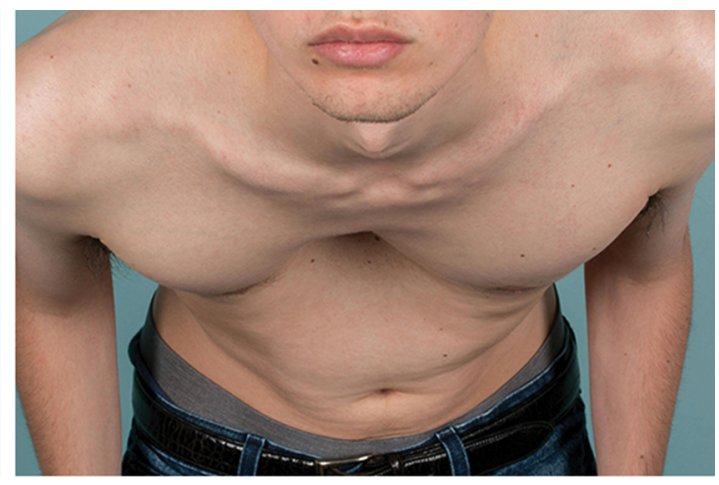

D

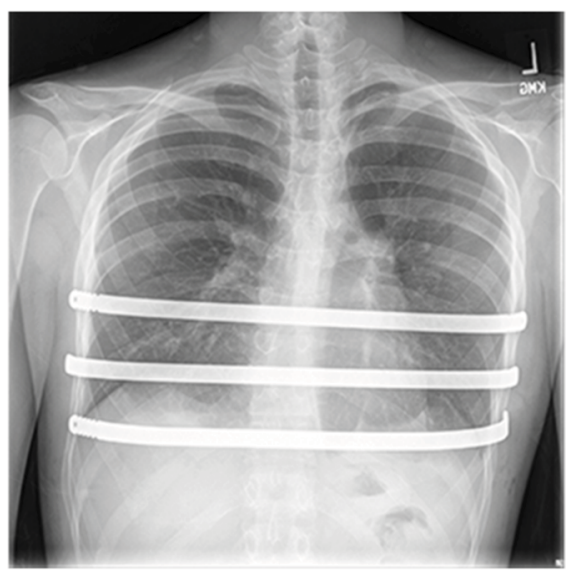

Figure 3 Clinical photographs of a 22-year-old man with severe pectus excavatum are shown before surgery (A, B) and after (C) minimally invasive repair of pectus excavatum, with placement of three Nuss bars as shown in the chest roentgenogram (D). 
Table 4 Review of several technical modifications reported for minimally invasive repair of pectus excavatum in adults

Technical modification
Forced sternal elevation:
- Crane technique
- Crane technique using
Kent retractor
- Two Langenbech hand held
retractors
- Horseshoe-shaped sternal elevator

- Vacuum bell

- Manual sternal lift and anchor

- Bone clamp and Rultract retractor

- T-fastener suture technique

\section{Bar stabilization techniques:}

- Five-point fixation

- Third point of fixation

- Bar flipping: Multipoint bar fixation; lateral sliding: insertion of stabilizer on the depressed side; hinge-point disruption: hinge point reinforcement.

- Three-point wire fixation

- Unilateral stabilizer and multiple polydioxanone (PDS) sutures around ribs

- Hinge plate

- Circumcostal sutures using Deschamps needle under endoscopic survey/lateral stabilizers

- FiberWire used to fix the bars circumferentially and bilaterally at multiple points

- Claw fixator (CFT) and hinge plate (HP)

- Bridge technique

- Unilateral stabilizer placed close to the hinge point, fixed to the bar by a steel wire

Study, years Reported results

- Park et al, ${ }^{61} 2015$

- Park et al, ${ }^{79} 2008$

- Yoon et al, ${ }^{69} 2010$

- Tedde et al, ${ }^{80} 2012$

- Takagi et al ${ }^{81}, 2012$

- Haecker et al, ${ }^{82} 2012$

- Johnson et al, ${ }^{83} 2013$

- Jaroszewski et al, ${ }^{78} 2014$

- Kim et al, ${ }^{84} 2014$

- Park et al, ${ }^{77} 2004$

- Hebra et al,,8 2006

- Park et al, ${ }^{79} 2008$

- Yoon et al, ${ }^{69} 2010$

- Kelly et al, ${ }^{51} 2010$

- Park et al, ${ }^{85} 201$ l

- Del Frari and Schwabegger, ${ }^{86}, 2014$

- McMahon et al, ${ }^{74} 2014$

- Park et al, 2015

- Pilegaard, ${ }^{75} 2015$
- Relieves pressure on the hinge points, thus preventing intercostal muscle stripping (type 3 bar displacement).

- Authors confirmed $0 \%$ intraoperative death and $0 \% 30$-day mortality.

- They observed that this manouver reduces the risk of pericardial sac and cardiac injury.

- No additional skin incision needed for insertion of the elevator, and it widens the retrosternal space for safer passage of thoracoscopically guided introducer.

- No cardiac, pericardial, or internal mammary vessel injuries were noted. Facilitates retrosternal dissection and bar insertion.

- Utilized even in patients with severe pectus excavatum (Haller index >7); $3 \mathrm{~cm}$ subxiphoid incision needed. Improves bar stability and reduces displacement. No intraoperative complications.

- Requires minimal additional incisions, decreases the force required to insert, rotates bars, and reduces the risk of intercostal muscle stripping in adult patients undergoing MIRPEx. The authors reported no intraoperative complications.

- Requires no specialized equipment, no incision in the anterior chest needed, does not cause any fracture or tear to the anterior chest structure. Disadvantage being removal of metal plate after positioning of the bar.

- All pericostal sutures can be done through the single tiny incision on each side, even in the parallel bar technique. Bar displacement: $3.4 \%$.

- Bar displacement: 6\%; stabilizer bar fracture: $3 \%$.

- Mechanism-based fixation effective in preventing bar displacement ( $4.6 \%$ vs $1.8 \%$ before and after MPF, respectively). Major complications decreased from $6.8 \%$ to $2 \%$ and reoperation rates decreased to $1.6 \%$ from $5.5 \%$ after MPF.

- Narrows the intercostal space, thereby preventing hinge point disruption, bar migration, and rotation. More effective in adults.

- Bar displacement requiring surgical repositioning decreased from $12 \%$ to I\%. Good to excellent anatomic result was obtained in $95.8 \%$.

- Bar displacement rate in patients without the hinge plate: $4 \%$ vs $0 \%$ in the hinge plate group. Hinge plate is effective in preventing an intercostal strip at hinge points and has a vital role in extending MIRPEx to adults.

- Prevents bar displacement. Excellent position of the bar with circumcostal sutures in $96 \%$, incomplete in $1.9 \%$, and poor in $1.9 \%$. With lateral stabilizers, $87.5 \%$ showed excellent position, and $12.5 \%$ showed poor position.

- Effective in preventing bar displacement and rotation. Metal stabilizers are not required.

- CFT used for sutureless bar fixation by hooking the rib with blades, whereas HP prevents intercostal muscle stripping at hinge points. Bar dislocation rate with CFT + HP: $0 \%$; reoperation rate: $3.38 \%$; total complication: $14.1 \%$. Authors recommend replacing conventional stabilizer with CFT and HP.

- Designed to connect two parallel bars using plates and screws to avoid bar displacement, with no use of sutures or invasive devices. During the follow-up, there was no virtual change in bar position, bar dislocation, or reoperation.

- No death, cardiac perforation, or deep infection occurred, and only $5 \%$ of patients experienced a complication. 
Table 4 (Continued)

\begin{tabular}{|c|c|c|}
\hline Technical modification & Study, years & Reported results \\
\hline $\begin{array}{l}\text { - Figure-of-eight FiberWire } \\
\text { reinforcement. Bars fixed bilaterally } \\
\text { and circumferentially around the rib } \\
\text { with FiberWire }\end{array}$ & - Jaroszewski et al, ${ }^{24} 2016$ & - Prevents lateral-posterior migration when stripping occurs. Bar rotation: $6.6 \%$. \\
\hline $\begin{array}{l}\text { - Stabilizer attached to bar with wire } \\
\text { or FiberWire suture on left with } \\
\text { multiple pericostal PDS sutures on } \\
\text { right }\end{array}$ & - Nuss et al, ${ }^{87} 2016$ & - Rate of displacement with stabilizers: $5 \%$, and with pericostal sutures: $1 \%$. \\
\hline \multicolumn{3}{|l|}{ Length of the bar: } \\
\hline $\begin{array}{l}\text { One inch }(2.5 \mathrm{~cm}) \text { shorter than the } \\
\text { measurement from right to left mid- } \\
\text { axillary line }\end{array}$ & - Kelly et al, ${ }^{51} 2010$ & $\begin{array}{l}\text { - Bar displacement requiring reoperation has been reduced from I3\% to } \\
1 \% \text {. }\end{array}$ \\
\hline $\begin{array}{l}\text { - Eleven inch }(7-15) \text { in } 200 \mathrm{I}-2010 \text { and } \\
10 \text { inch }(8-14) \text { in } 201 \mathrm{I}-2016\end{array}$ & - Pilegaard, ${ }^{55} 2016$ & $\begin{array}{l}\text { - Reported lower rate of bar malrotations, and surgery can be done in less } \\
\text { than an hour for over } 90 \% \text { of cases. ( }<2 \% \text { bars flipped) }\end{array}$ \\
\hline \multicolumn{3}{|l|}{ Multiple bars: } \\
\hline - Two bars & - Nuss, ${ }^{88} 2008$ & - Bar displacement: 5\%; requiring revision: $50 \%$ \\
\hline - Two bars in $32 \%$ & $\begin{array}{l}\text { - Pilegaard and Licht, } \\
2008\end{array}$ & $\begin{array}{l}\text { - Use of multiple bars was significantly more common }(P<0.0 \text { I }) \text { in adults } \\
\text { compared to younger patients, where } 86 \% \text { received one bar and I } 4 \% \\
\text { needed two bars. }\end{array}$ \\
\hline - Double-bar application & - Nagaso et al, ${ }^{89} 2010$ & $\begin{array}{l}\text { Patients in one-bar group required self-injection of intravenous narcotics } \\
\text { more frequently than patients in double-bar group (double-bar decreased } \\
\text { postoperative pain). Stresses on the thoraces were smaller with double } \\
\text { bars than with a single bar. }\end{array}$ \\
\hline - Two bars & - Stanfill et al, ${ }^{90} 2012$ & $\begin{array}{l}\text { - No patient required revision for bar displacement when two bars were } \\
\text { used as opposed to } 15.5 \% \text { who required reoperation for bar movement } \\
\text { when one bar was initially placed. }(P=0.05) \text {, with no difference in patient } \\
\text { age and Haller index between groups. }\end{array}$ \\
\hline - Three bars & - Jaroszewski et al, ${ }^{24} 2016$ & $\begin{array}{l}\text { - More than } 40 \% \text { of patients of both adults over } 30 \text { year old and patients } \\
\text { between } 18-29 \text { years groups required three bars }\end{array}$ \\
\hline \multicolumn{3}{|l|}{ Hybrid approach/osteotomy: } \\
\hline $\begin{array}{l}\text { - Transverse sternotomy/limited } \\
\text { sternal resection/parasternal bar } \\
\text { fixation }\end{array}$ & - Dzielicki et al, 2006 & $\begin{array}{l}\text { - Further procedures were essential to achieve and maintain an adequate } \\
\text { correction and to decrease sternal rigidity and its pressure on the bar. }\end{array}$ \\
\hline - MOVARPE technique & $\begin{array}{l}\text { - Del Frari and } \\
\text { Schwabegger }{ }^{86}, 2014\end{array}$ & $\begin{array}{l}\text { - Used in adults with athletic disposition, deformities with deep funnel, and } \\
\text { severe asymmetry. Only minor complications (4.4\%) were observed. }\end{array}$ \\
\hline - Scoring of deformed cartilages & - Nagasao et al, ${ }^{22} 2016$ & $\begin{array}{l}\text { - Postoperative pain as measured by the frequency of administration of } \\
\text { anesthetics for } 2 \text { days was reduced: } 4.9 \text { vs } 2.5 \text {. }\end{array}$ \\
\hline - Hybrid approach & - Jaroszewski et al, ${ }^{24} 2016$ & $\begin{array}{l}\text { - Open-cartilage resection, sternal osteotomy, or both was more } \\
\text { commonly performed in patients older than } 30 \text { years (mean, } 47.8 \text { years vs } \\
39.5 \text { years; } P=0.0003 \text { ) and with defect severity ( } 11.3 \% \text { vs } 3.5 \% \text { in younger } \\
\text { patients). }\end{array}$ \\
\hline
\end{tabular}

Abbreviations: MIRPEx, minimally invasive repair of pectus excavatum; MPF, multipoint pericostal bar fixation; MOVARPE, minor open videoendoscopic assisted repair of pectus excavatum.

Nuss revision after procedure failure, too long bars were noted to be a factor related to failure. ${ }^{95}$

The biggest challenge in adult patients continues to be bar fixation. A higher rate of bar displacement is reported in older patients. ${ }^{14,24}$ There are multiple successful ways reported for securing of bars. Medial fixation with a hinge reinforcement plate, ${ }^{85}$ medially placed stabilizers ${ }^{75}$ multipoint fixation, ${ }^{24,69,77}$ and the Bridge technique, which was more recently published, ${ }^{61}$ have all been successful methods for bar fixation in adult patients. $72,79,87,96$
Chondroplasty or open osteotomy may still be necessary to achieve adequate repair in some adult patients. Patients with complex combined deformities, extensively calcified chest walls, and significant asymmetry may require an open repair for optimal correction. The requirement for osteotomy or cartilage resection is more commonly reported in older patients..$^{24,91,97,98}$ In our experience, over $88 \%$ of the patients $\geq 30$ years were successfully repaired with MIRPEx; however, some required an osteotomy or open resection for fracture. Postoperative pain may also be reduced by scoring 


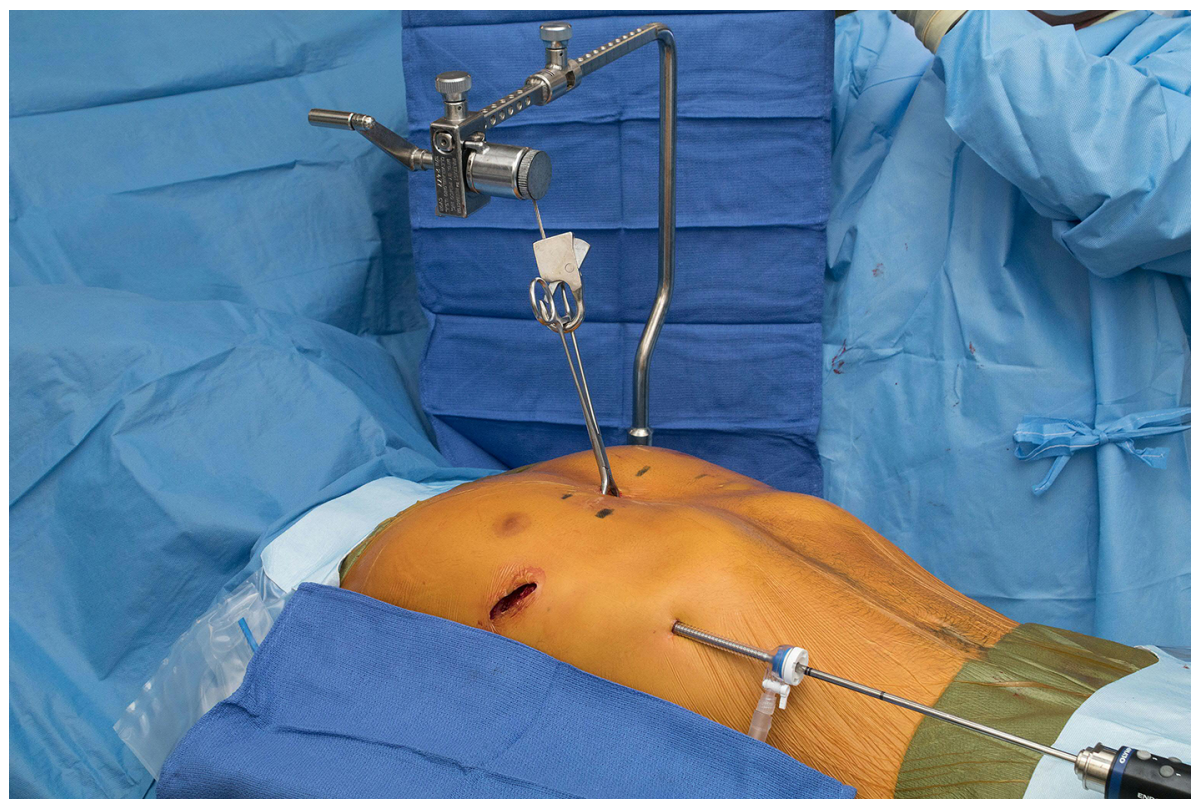

Figure 4 The Rultract retractor can be utilized to forcefully elevate the sternum when attached by a bone clamp.

of deformed cartilages as illustrated by Nagasao et al. ${ }^{92}$ The use of a hybrid procedure may also be considered and is our procedure of choice for these more difficult deformities. ${ }^{24}$ Both surgical principles are utilized by incorporating osteotomy cuts and external fixation as well as pectus support bars. Achieving adequate postoperative pain control remains a concern for adults undergoing Nuss. ${ }^{99}$ Various analgesic regimens have been discussed by several authors. ${ }^{100}$ Perioperative pain can be well managed by current techniques. ${ }^{101}$ These include the use of thoracic epidurals, intravenous ondemand patient-administrated narcotics, local paravertebral blocks, and subcutaneous continuous flow catheters. ${ }^{102-106} \mathrm{We}$ have had excellent results using a protocol including gabapentin, ibuprofen, acetaminophen, and narcotics along with subcutaneous continuous flow catheters for postoperative pain control. ${ }^{24,102,107}$ Adjuvant medications for postoperative pain management have included the use of ketorolac, diazepam, and gabapentin. ${ }^{103-106}$ Intraoperative use of methadone can also be advantageous. ${ }^{24,102}$

\section{Discussion}

The extension of the Nuss procedure to repair adults with PEx has been controversial in the past. ${ }^{14,65-67}$ There are now multiple publications that report successful repair of adults even beyond 50 years of age. ${ }^{912,24,55}$ The difficulty of repair and risk of complications do, however, increase with age. ${ }^{14,24,53}$ Adequate surgical experience with the Nuss procedure in younger patients that are easier to repair is critical prior to attempting the more difficult adult deformity.
Bar rotation and migration can be a significant issue and techniques to minimize intercostal stripping, such as reinforcement of intercostal spaces ${ }^{24,59,87}$ and medially placed stabilizers, may be of benefit in reducing the risks. ${ }^{75}$ The use of forced sternal elevation can also decrease the forces required for bar insertion and positioning. ${ }^{69,78-84}$ The adult chest wall has additional complexities due to the decrease in flexibility and increase in weight. Multiple bars have been noted to decrease the weight supported by an individual bar and decrease the risk of rotation. ${ }^{24,70,88-90}$ Adequate stabilization of bars is also critical due to these factors, and medial and/or multipoint fixation has been shown to reduce bar displacement. ${ }^{24,28,51,59,61,69,75,86,87}$ We did not intend this publication to be an intensive review of surgical techniques in adult patients, and the majority of information presented was based on a larger case series which reported on primary Nuss repair in the adult population. Extension of the Nuss procedure to more complex repairs, such as patients with prior sternotomy or cardiac surgery, is beyond the scope of this paper and can be associated with catastrophic complications. ${ }^{55,108}$

\section{Conclusion}

MIRPEx can be extended to repair the majority of older adult patients. Although adults undergoing Nuss procedure may have a higher rate of complications, continuous technical refinements have significantly reduced the complication rates and contributed to the success of the procedure. As there is increased difficulty in performing this procedure 
in adult patients, the experience and expertise of surgeons at specialized centers is critical for successful outcomes. There is enough evidence to validate repair of adults with PEx. Published data support the benefits of repair with good outcomes and improvement of symptoms.

\section{Disclosure}

The authors report no conflicts of interest in this work.

\section{References}

1. Fokin AA, Steuerwald NM, Ahrens WA, Allen KE. Anatomical, histologic, and genetic characteristics of congenital chest wall deformities. Semin Thorac Cardiovasc Surg. 2009;21(1):44-57.

2. Scherer LR, Arn PH, Dressel DA, Pyeritz RM, Haller JA, Jr. Surgical management of children and young adults with Marfan syndrome and pectus excavatum. J Pediatr Surg. 1988;23(12):1169-1172.

3. Cobben JM, Oostra RJ, van Dijk FS. Pectus excavatum and carinatum. Eur J Med Genet. 2014;57(8):414-417.

4. Chung CS, Myrianthopoulos NC. Factors affecting risks of congenital malformations. I. Analysis of epidemiologic factors in congenital malformations. Report from the Collaborative Perinatal Project. Birth Defects Orig Artic Ser. 1975;11(10):1-22.

5. Fonkalsrud EW. Management of pectus chest deformities in female patients. Am J Surg. 2004;187(2):192-197.

6. Ma IT, Rebecca AM, Notrica DM, McMahon LE, Jaroszewski DE. Pectus excavatum in adult women: repair and the impact of prior or concurrent breast augmentation. Plast Reconstr Surg. 2015;135(2): $303 \mathrm{e}-312 \mathrm{e}$.

7. Park HJ, Gu JH, Jang JC, Dhong ES, Yoon ES. Correction of pectus excavatum with breast hypoplasia using simultaneous pectus bar procedure and augmentation mammoplasty. Ann Plast Surg. 2014;73(2):190-195.

8. Sigalet DL, Montgomery M, Harder J, Wong V, Kravarusic D, Alassiri A. Long term cardiopulmonary effects of closed repair of pectus excavatum. Pediatr Surg Int. 2007;23(5):493-497.

9. Chao CJ, Jaroszewski DE, Kumar PN, et al. Surgical repair of pectus excavatum relieves right heart chamber compression and improves cardiac output in adult patients - an intraoperative transesophageal echocardiographic study. Am J Surg. 2015;210(6):1118-1124.

10. Kelly RE, Jr., Cash TF, Shamberger RC, et al. Surgical repair of pectus excavatum markedly improves body image and perceived ability for physical activity: multicenter study. Pediatrics. 2008;122(6): 1218-1222.

11. Jaroszewski DE, Fonkalsrud EW. Repair of pectus chest deformities in 320 adult patients: 21 year experience. Ann Thorac Surg. 2007;84(2):429-433.

12. Kragten HA, Siebenga J, Hoppener PF, Verburg R, Visker N. Symptomatic pectus excavatum in seniors (SPES): a cardiovascular problem?: a prospective cardiological study of 42 senior patients with a symptomatic pectus excavatum. Neth Heart J. 2011;19(2):73-78.

13. Jaroszewski D, Steidley E, Galindo A, Arabia F. Treating heart failure and dyspnea in a 78-year-old man with surgical correction of pectus excavatum. Ann Thorac Surg. 2009;88(3):1008-1010.

14. Kim do H, Hwang JJ, Lee MK, Lee DY, Paik HC. Analysis of the Nuss procedure for pectus excavatum in different age groups. Ann Thorac Surg. 2005;80(3):1073-1077.

15. Esteves E, Paiva KC, Calcagno-Silva M, Chagas CC, Barbosa-Filho $\mathrm{H}$. Treatment of pectus excavatum in patients over 20 years of age. $J$ Laparoendosc Adv Surg Tech A. 2011;21(1):93-96.

16. Kaguraoka H, Ohnuki T, Itaoka T, Kei J, Yokoyama M, Nitta S. Degree of severity of pectus excavatum and pulmonary function in preoperative and postoperative periods. J Thorac Cardiovasc Surg. 1992;104(5):1483-1488.
17. Morshuis WJ, Folgering HT, Barentsz JO, Cox AL, van Lier HJ, Lacquet LK. Exercise cardiorespiratory function before and one year after operation for pectus excavatum. J Thorac Cardiovasc Surg. 1994;107(6):1403-1409.

18. Haller JA Jr, Loughlin GM. Cardiorespiratory function is significantly improved following corrective surgery for severe pectus excavatum. Proposed treatment guidelines. J Cardiovasc Surg (Torino). 2000;41(1):125-130.

19. Neviere R, Montaigne D, Benhamed L, et al. Cardiopulmonary response following surgical repair of pectus excavatum in adult patients. Eur J Cardiothorac Surg. 2011;40(2):e77-e82.

20. Malek MH, Fonkalsrud EW, Cooper CB. Ventilatory and cardiovascular responses to exercise in patients with pectus excavatum. Chest. 2003;124(3):870-882.

21. Gurkan U, Aydemir B, Aksoy S, et al. Echocardiographic assessment of right ventricular function before and after surgery in patients with pectus excavatum and right ventricular compression. Thorac Cardiovasc Surg. 2014;62(3):231-235.

22. Coln E, Carrasco J, Coln D. Demonstrating relief of cardiac compression with the Nuss minimally invasive repair for pectus excavatum. J Pediatr Surg. 2006;41(4):683-686; discussion 683-686.

23. Mocchegiani R, Badano L, Lestuzzi C, Nicolosi GL, Zanuttini D. Relation of right ventricular morphology and function in pectus excavatum to the severity of the chest wall deformity. Am J Cardiol. 1995;76(12):941-946.

24. Jaroszewski DE, Ewais MM, Chao CJ, et al. Success of minimally invasive pectus excavatum procedures (modified Nuss) in adult patients ( $\pm=30$ years). Ann Thorac Surg. 2016;102(3):993-1003.

25. Krueger T, Chassot PG, Christodoulou M, Cheng C, Ris HB, Magnusson L. Cardiac function assessed by transesophageal echocardiography during pectus excavatum repair. Ann Thorac Surg. 2010;89(1):240-243.

26. Neviere R, Benhamed L, Duva Pentiah A, Wurtz A. Pectus excavatum repair improves respiratory pump efficacy and cardiovascular function at exercise. J Thorac Cardiovasc Surg. 2013;145(2):605-606.

27. Kelly RE Jr, Mellins RB, Shamberger RC, et al. Multicenter study of pectus excavatum, final report: complications, static/exercise pulmonary function, and anatomic outcomes. J Am Coll Surg. 2013;217(6):1080-1089.

28. Hebra A, Jacobs JP, Feliz A, Arenas J, Moore CB, Larson S. Minimally invasive repair of pectus excavatum in adult patients. Am Surg. 2006;72(9):837-842.

29. Aronson DC, Bosgraaf RP, van der Horst C, Ekkelkamp S. Nuss procedure: pediatric surgical solution for adults with pectus excavatum. World J Surg. 2007;31(1):26-29; discussion 30.

30. Cheng YL, Lee SC, Huang TW, Wu CT. Efficacy and safety of modified bilateral thoracoscopy-assisted Nuss procedure in adult patients with pectus excavatum. Eur J Cardiothorac Surg. 2008;34(5): 1057-1061.

31. Udholm S, Maagaard M, Pilegaard H, Hjortdal V. Cardiac function in adults following minimally invasive repair of pectus excavatum. Interact Cardiovasc Thorac Surg. 2016;22(5):525-529.

32. Topper A, Polleichtner S, Zagrosek A, et al. Impact of surgical correction of pectus excavatum on cardiac function: insights on the right ventricle. A cardiovascular magnetic resonance studydagger. Interact Cardiovasc Thorac Surg. 2016;22(1):38-46.

33. Albouaini K, Egred M, Alahmar A, Wright DJ. Cardiopulmonary exercise testing and its application. Heart. 2007;93(10):1285-1292.

34. Malek MH, Coburn JW. Strategies for cardiopulmonary exercise testing of pectus excavatum patients. Clinics (Sao Paulo). 2008;63(2):245-254.

35. Maagaard M, Tang M, Ringgaard S, et al. Normalized cardiopulmonary exercise function in patients with pectus excavatum three years after operation. Ann Thorac Surg. 2013;96(1):272-278.

36. Perhonen MA, Franco F, Lane LD, et al. Cardiac atrophy after bed rest and spaceflight. J Appl Physiol (1985). 2001;91(2):645-653. 
37. O'Keefe J, Byrne R, Montgomery M, Harder J, Roberts D, Sigalet DL. Longer term effects of closed repair of pectus excavatum on cardiopulmonary status. J Pediatr Surg. 2013;48(5):1049-1054.

38. Tang M, Nielsen HH, Lesbo $\mathrm{M}$, et al. Improved cardiopulmonary exercise function after modified Nuss operation for pectus excavatum. Eur J Cardiothorac Surg. 2012;41(5):1063-1067.

39. Bawazir OA, Montgomery M, Harder J, Sigalet DL. Midterm evaluation of cardiopulmonary effects of closed repair for pectus excavatum. J Pediatr Surg. 2005;40(5):863-867.

40. Jaroszewski D, Notrica D, McMahon L, Steidley DE, Deschamps C. Current management of pectus excavatum: a review and update of therapy and treatment recommendations. J Am Board Fam Med. 2010;23(2):230-239.

41. Hanna WC, Ko MA, Blitz M, Shargall Y, Compeau CG. Thoracoscopic Nuss procedure for young adults with pectus excavatum: excellent midterm results and patient satisfaction. Ann Thorac Surg. 2013;96(3):1033-1036; discussion 1037-1038.

42. Jacobsen EB, Thastum M, Jeppesen JH, Pilegaard HK. Health-related quality of life in children and adolescents undergoing surgery for pectus excavatum. Eur J Pediatr Surg. 2010;20(2):85-91.

43. Krasopoulos G, Dusmet M, Ladas G, Goldstraw P. Nuss procedure improves the quality of life in young male adults with pectus excavatum deformity. Eur J Cardiothorac Surg. 2006;29(1):1-5.

44. Kuru P, Bostanci K, Ermerak NO, Bahadir AT, Afacan C, Yuksel M. Quality of life improves after minimally invasive repair of pectus excavatum. Asian Cardiovasc Thorac Ann. 2015;23(3):302-307.

45. Steinmann C, Krille S, Mueller A, Weber P, Reingruber B, Martin A. Pectus excavatum and pectus carinatum patients suffer from lower quality of life and impaired body image: a control group comparison of psychological characteristics prior to surgical correction. Eur $J$ Cardiothorac Surg. 2011;40(5):1138-1145.

46. Kelly RE, Jr. Pectus excavatum: historical background, clinical picture, preoperative evaluation and criteria for operation. Semin Pediatr Surg. 2008;17(3):181-193.

47. Krasopoulos G, Goldstraw P. Minimally invasive repair of pectus excavatum deformity. Eur J Cardiothorac Surg. 2011;39(2): 149-158.

48. Metzelder ML, Kuebler JF, Leonhardt J, Ure BM, Petersen C. Self and parental assessment after minimally invasive repair of pectus excavatum: lasting satisfaction after bar removal. Ann Thorac Surg. 2007;83(5):1844-1849.

49. Lomholt JJ, Jacobsen EB, Thastum M, Pilegaard H. A prospective study on quality of life in youths after pectus excavatum correction. Ann Cardiothorac Surg. 2016;5(5):456-465.

50. Hoksch B, Kocher G, Vollmar P, Praz F, Schmid RA. Nuss procedure for pectus excavatum in adults: long-term results in a prospective observational study. Eur J Cardiothorac Surg. 2016;50(5):934-939.

51. Kelly RE, Goretsky MJ, Obermeyer R, et al. Twenty-one years of experience with minimally invasive repair of pectus excavatum by the Nuss procedure in 1215 patients. Ann Surg. 2010;252(6):1072-1081.

52. Tikka T, Kalkat MS, Bishay E, Steyn RS, Rajesh PB, Naidu B. A 20-year review of pectus surgery: an analysis of factors predictive of recurrence and outcomes. Interact Cardiovasc Thorac Surg. 2016;23(6):908-913.

53. Sacco Casamassima MG, Gause C, Goldstein SD, et al. Patient satisfaction after minimally invasive repair of pectus excavatum in adults: long-term results of Nuss procedure in adults. Ann Thorac Surg. 2016;101(4):1338-1345.

54. Tikka T, Webb J, Agostini P, et al. Pectus patient information website has improved access to care and patient reported outcomes. J Cardiothorac Surg. 2016;11(1):69.

55. Pilegaard HK. Single centre experience on short bar technique for pectus excavatum. Ann Cardiothorac Surg. 2016;5(5):450-455.

56. Pawlak K, Gasiorowski L, Gabryel P, Galecki B, Zielinski P, Dyszkiewicz W. Early and late results of the Nuss procedure in surgical treatment of pectus excavatum in different age groups. Ann Thorac Surg. 2016;102(5):1711-1716.
57. Ersen E, Demirkaya A, Kilic B, et al. Minimally invasive repair of pectus excavatum (MIRPE) in adults: is it a proper choice? Wideochir Inne Tech Maloinwazyjne. 2016;11(2):98-104.

58. Fibla JJ, Molins L, Moradiellos J, et al. Experience with the Nuss technique for the treatment of pectus excavatum in Spanish thoracic surgery departments. Cir Esp. 2016;94(1):38-43.

59. Park HJ, Kim KS, Lee S, Jeon HW. A next-generation pectus excavatum repair technique: new devices make a difference. Ann Thorac Surg. 2015;99(2):455-461

60. Zhang DK, Tang JM, Ben XS, et al. Surgical correction of 639 pectus excavatum cases via the Nuss procedure. J Thorac Dis. 2015;7(9):1595-1605.

61. Park HJ, Kim KS, Moon YK, Lee S. The bridge technique for pectus bar fixation: a method to make the bar un-rotatable. J Pediatr Surg. 2015;50(8):1320-1322.

62. Rokitansky AM, Stanek R. Modified minimally invasive pectus repair in children, adolescents and adults: an analysis of 262 patients. Pneumologia. 2013;62(4):224-231.

63. Olbrecht VA, Arnold MA, Nabaweesi R, et al. Lorenz bar repair of pectus excavatum in the adult population: should it be done? Ann Thorac Surg. 2008;86(2):402-408; discussion 408-409.

64. Nuss D, Kelly RE Jr, Croitoru DP, Katz ME. A 10-year review of a minimally invasive technique for the correction of pectus excavatum. J Pediatr Surg. 1998;33(4):545-552.

65. Johnson WR, Fedor D, Singhal S. Systematic review of surgical treatment techniques for adult and pediatric patients with pectus excavatum. J Cardiothorac Surg. 2014;9:25.

66. Hebra A, Swoveland B, Egbert M, et al. Outcome analysis of minimally invasive repair of pectus excavatum: review of 251 cases. $J$ Pediatr Surg. 2000;35(2):252-257; discussion 257-258.

67. Kanagaratnam A, Phan S, Tchantchaleishvili V, Phan K. Ravitch versus Nuss procedure for pectus excavatum: systematic review and meta-analysis. Ann Cardiothorac Surg. 2016;5(5):409-421.

68. Pilegaard HK. Extending the use of Nuss procedure in patients older than 30 years. Eur J Cardiothorac Surg. 2011;40(2):334-337.

69. Yoon YS, Kim HK, Choi YS, Kim K, Shim YM, Kim J. A modified Nuss procedure for late adolescent and adult pectus excavatum. World J Surg. 2010;34(7):1475-1480.

70. Pilegaard HK, Licht PB. Routine use of minimally invasive surgery for pectus excavatum in adults. Ann Thorac Surg. 2008;86(3):952-956.

71. Schalamon J, Pokall S, Windhaber J, Hoellwarth ME. Minimally invasive correction of pectus excavatum in adult patients. $J$ Thorac Cardiovasc Surg. 2006;132(3):524-529.

72. Park HJ, Jeong JY, Jo WM, et al. Minimally invasive repair of pectus excavatum: a novel morphology-tailored, patient-specific approach. J Thorac Cardiovasc Surg. 2010;139(2):379-386.

73. Mansour KA, Thourani VH, Odessey EA, Durham MM, Miller JI Jr, Miller DL. Thirty-year experience with repair of pectus deformities in adults. Ann Thorac Surg. 2003;76(2):391-395; discussion 395.

74. McMahon LE, Johnson KN, Jaroszewski DE, et al. Experience with FiberWire for pectus bar attachment. J Pediatr Surg. 2014;49(8): 1259-1263.

75. Pilegaard HK. Nuss technique in pectus excavatum: a mono-institutional experience. J Thorac Dis. 2015;7 (Suppl 2):S172-S176.

76. Molik KA, Engum SA, Rescorla FJ, West KW, Scherer LR, Grosfeld JL. Pectus excavatum repair: experience with standard and minimal invasive techniques. J Pediatr Surg. 2001;36(2):324-328.

77. Park HJ, Lee SY, Lee CS, Youm W, Lee KR. The Nuss procedure for pectus excavatum: evolution of techniques and early results on 322 patients. Ann Thorac Surg. 2004;77(1):289-295.

78. Jaroszewski DE, Johnson K, McMahon L, Notrica D. Sternal elevation before passing bars: a technique for improving visualization and facilitating minimally invasive pectus excavatum repair in adult patients. J Thorac Cardiovasc Surg. 2014;147(3):1093-1095.

79. Park HJ, Chung WJ, Lee IS, Kim KT. Mechanism of bar displacement and corresponding bar fixation techniques in minimally invasive repair of pectus excavatum. J Pediatr Surg. 2008;43(1):74-78. 
80. Tedde ML, de Campos JR, Wihlm JM, Jatene FB. The Nuss procedure made safer: an effective and simple sternal elevation manoeuvre. Eur J Cardiothorac Surg. 2012;42(5):890-891.

81. Takagi S, Oyama T, Tomokazu N, Kinoshita K, Makino T, Ohjimi H. A new sternum elevator reduces severe complications during minimally invasive repair of the pectus excavatum. Pediatr Surg Int. 2012;28(6):623-626.

82. Haecker FM, Sesia SB. Intraoperative use of the vacuum bell for elevating the sternum during the Nuss procedure. J Laparoendosc Adv Surg Tech A. 2012;22(9):934-936.

83. Johnson WR, Fedor D, Singhal S. A novel approach to eliminate cardiac perforation in the nuss procedure. Ann Thorac Surg. 2013;95(3):1109-1111.

84. Kim D, Idowu O, Palmer B, Kim S. Anterior chest wall elevation using a T-fastener suture technique during a nuss procedure. Ann Thorac Surg. 2014;98(2):734-736.

85. Park HJ, Jeong JY, Kim KT, Choi YH. Hinge reinforcement plate for adult pectus excavatum repair: a novel tool for the prevention of intercostal muscle strip. Interact Cardiovasc Thorac Surg. 2011;12(5):687-691.

86. Del Frari B, Schwabegger AH. How to avoid pectus bar dislocation in the MIRPE or MOVARPE technique: results of 12 years' experience. Ann Plast Surg. 2014;72(1):75-79.

87. Nuss D, Obermeyer RJ, Kelly RE. Nuss bar procedure: past, present and future. Ann Cardiothorac Surg. 2016;5(5):422-433.

88. Nuss D. Minimally invasive surgical repair of pectus excavatum. Semin Pediatr Surg. 2008;17(3):209-217.

89. Nagaso T, Miyamoto J, Kokaji K, et al. Double-bar application decreases postoperative pain after the Nuss procedure. $J$ Thorac Cardiovasc Surg. 2010;140(1):39-44, 44.e1-2.

90. Stanfill AB, DiSomma N, Henriques SM, Wallace LJ, Vegunta RK, Pearl RH. Nuss procedure: decrease in bar movement requiring reoperation with primary placement of two bars. J Laparoendosc Adv Surg Tech A. 2012;22(4):412-415.

91. Dzielicki J, Korlacki W, Janicka I, Dzielicka E. Difficulties and limitations in minimally invasive repair of pectus excavatum -6 years experiences with Nuss technique. Eur J Cardiothorac Surg. 2006;30(5):801-804.

92. Nagasao T, Hamamoto Y, Tamai M, et al. Scoring of deformed costal cartilages reduces postoperative pain after Nuss procedure for pectus excavatum. Thorac Cardiovasc Surg. 2016;64(1):62-69.

93. Pilegaard HK. Short Nuss bar procedure. Ann Cardiothorac Surg. 2016;5(5):513-518.

94. Ghionzoli M, Ciuti G, Ricotti L, et al. Is a shorter bar an effective solution to avoid bar dislocation in a Nuss procedure? Ann Thorac Surg. 2014;97(3):1022-1027.

95. Croitoru DP, Kelly RE Jr, Goretsky MJ, Gustin T, Keever R, Nuss D. The minimally invasive Nuss technique for recurrent or failed pectus excavatum repair in 50 patients. J Pediatr Surg. 2005;40(1):181-186; discussion 186-187.
96. Fallon SC, Slater BJ, Nuchtern JG, et al. Complications related to the Nuss procedure: minimizing risk with operative technique. J Pediatr Surg. 2013;48(5):1044-1048.

97. Al-Assiri A, Kravarusic D, Wong V, Dicken B, Milbrandt K, Sigalet DL. Operative innovation to the "Nuss" procedure for pectus excavatum: operative and functional effects. J Pediatr Surg. 2009;44(5): 888-892.

98. Ravenni G, Actis Dato GM, Zingarelli E, Flocco R, Casabona R. Nuss procedure in adult pectus excavatum: a simple artifice to reduce sternal tension. Interact Cardiovasc Thorac Surg. 2013;17(1):23-25.

99. Papic JC, Finnell SM, Howenstein AM, Breckler F, Leys CM. Postoperative opioid analgesic use after Nuss versus Ravitch pectus excavatum repair. J Pediatr Surg. 2014;49(6):919-923; discussion 923.

100. Muhly WT, Maxwell LG, Cravero JP. Pain management following the Nuss procedure: a survey of practice and review. Acta Anaesthesiol Scand. 2014;58(9):1134-1139.

101. Kelly RE, Jr., Shamberger RC, Mellins RB, et al. Prospective multicenter study of surgical correction of pectus excavatum: design, perioperative complications, pain, and baseline pulmonary function facilitated by internet-based data collection. J Am Coll Surg. 2007;205(2):205-216.

102. Jaroszewski DE, Temkit M, Ewais MM, et al. Randomized trial of epidural vs. subcutaneous catheters for managing pain after modified Nuss in adults. J Thorac Dis. 2016;8(8):2102-2110.

103. Gebhardt R, Mehran RJ, Soliz J, Cata JP, Smallwood AK, Feeley TW. Epidural versus ON-Q local anesthetic-infiltrating catheter for post-thoracotomy pain control. J Cardiothorac Vasc Anesth. 2013;27(3):423-426.

104. Ried M, Schilling C, Potzger T, et al. Prospective, comparative study of the On-Q(R) PainBuster(R) postoperative pain relief system and thoracic epidural analgesia after thoracic surgery. J Cardiothorac Vasc Anesth. 2014;28(4):985-990.

105. Weber T, Matzl J, Rokitansky A, Klimscha W, Neumann K, Deusch E. Superior postoperative pain relief with thoracic epidural analgesia versus intravenous patient-controlled analgesia after minimally invasive pectus excavatum repair. JThorac Cardiovasc Surg. 2007;134(4): 865-870.

106. Futagawa K, Suwa I, Okuda T, et al. Anesthetic management for the minimally invasive Nuss procedure in 21 patients with pectus excavatum. J Anesth. 2006;20(1):48-50.

107. Hall Burton DM, Boretsky KR. A comparison of paravertebral nerve block catheters and thoracic epidural catheters for postoperative analgesia following the Nuss procedure for pectus excavatum repair. Paediatr Anaesth. 2014;24(5):516-520.

108. Jaroszewski DE, Gustin PJ, Haecker F-M, et al. Pectus excavatum repair after sternotomy: the Chest Wall International Group experience with substernal Nuss bars. European Journal of Cardio-Thoracic Surgery. 2017;52(4):710-717.
Patient Related Outcome Measures

\section{Publish your work in this journal}

Patient Related Outcome Measures is an international, peer-reviewed, open access journal focusing on treatment outcomes specifically relevant to patients. All aspects of patient care are addressed within the journal and practitioners from all disciplines are invited to submit their work as well as healthcare researchers and patient support groups.
Dovepress

The journal is included in PubMed. The manuscript management system is completely online and includes a very quick and fair peer-review system. Visit http://www.dovepress.com/testimonials.php to read real quotes from published authors. 